\title{
Integrated Radiation Transport and Nuclear Fuel Performance for Assembly-Level Simulations
}

\section{January 2012}

Prepared by

Kevin Clarno

Steven Hamilton

Bobby Philip

Mark Berrill

Rahul Sampath

Srikanth Allu

David Pugmire

Gary Dilts, Los Alamos National Laboratory James Banfield, University of Tennessee-Knoxville Thomas Evans

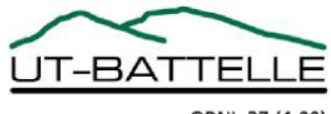




\section{DOCUMENT AVAILABILITY}

Reports produced after January 1, 1996, are generally available free via the U.S. Department of Energy (DOE) Information Bridge:

Web Site: http://www.osti.gov/bridge

Reports produced before January 1, 1996, may be purchased by members of the public from the following source:

National Technical Information Service

5285 Port Royal Road

Springfield, VA 22161

Telephone: 703-605-6000 (1-800-553-6847)

TDD: $703-487-4639$

Fax: 703-605-6900

E-mail:info@ntis.fedworld.gov

Web site: http://www.ntis.gov/support/ordernowabout.htm

Reports are available to DOE employees, DOE contractors, Energy Technology Data Exchange (ETDE), and International Nuclear Information System (INIS) representatives from the following sources:

Office of Scientific and Technical Information

P.O. Box 62

Oak Ridge, TN 37831

Telephone: 865-576-8401

Fax: 865-576-5728

E-mail: report@osti.gov

Web site:http://wwW.osti.gov/contact.html

This report was prepared as an account of work sponsored by an agency of the United States Government. Neither the United States nor any agency thereof, nor any of their employees, makes any warranty, express or implied, or assumes any legal liability or responsibility for the accuracy, completeness, or usefulness of any information, apparatus, product, or process disclosed, or represents that its use would not infringe privately owned rights. Reference herein to any specific commercial product, process, or service by trade name, trademark, manufacturer, or otherwise, does not necessarily constitute or imply its endorsement, recommendation, or favoring by the United States Government or any agency thereof. The views and opinions of authors expressed herein do not necessarily state or reflect those of the United States Government or any agency thereof. 
Reactor and Nuclear Systems Division

\title{
INTEGRATED RADIATION TRANSPORT AND NUCLEAR FUEL PERFORMANCE FOR ASSEMBLY-LEVEL SIMULATIONS
}

\author{
Kevin Clarno \\ Steven Hamilton \\ Bobby Philip \\ Mark Berrill \\ Rahul Sampath \\ Srikanth Allu \\ David Pugmire \\ Gary Dilts, Los Alamos National Laboratory \\ James Banfield, University of Tennessee-Knoxville \\ Thomas Evans
}

Date Published: January 2012

\author{
Prepared by \\ OAK RIDGE NATIONAL LABORATORY \\ P.O. Box 2008 \\ Oak Ridge, Tennessee 37831-6285 \\ managed by \\ UT-Battelle, LLC \\ for the \\ U.S. DEPARTMENT OF ENERGY \\ under contract DE-AC05-00OR22725
}





\section{Contents}

LIST OF FIGURES $\ldots \ldots \ldots \ldots \ldots \ldots \ldots \ldots \ldots \ldots \ldots \ldots$

LIST OF TABLES $\ldots \ldots \ldots \ldots \ldots \ldots \ldots \ldots \ldots \ldots \ldots \ldots$ vii

ABSTRACT . . . . . . . . . . . . . . . . . . . . . . . 1

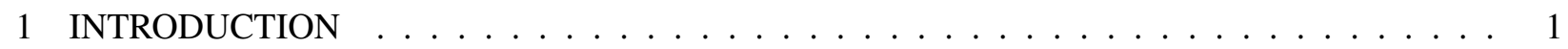

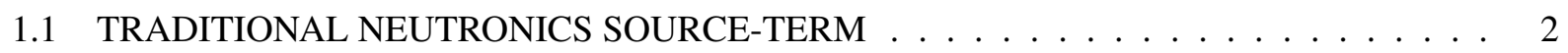

1.2 POTENTIAL APPLICATIONS OF THE INTEGRATED CAPABILITY . . . . . . . . . . . 3

$1.2 .1 \quad$ Fuel Failures in the Hatch Nuclear Power Plant . . . . . . . . . . . . . . . . 3

1.2 .2 Shutdown Heat Removal Tests in the Experimental Breeder Reactor - II . . . . . . . 3

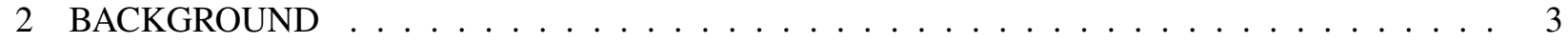

2.1 DEFINITION OF THE PROBLEM $\ldots \ldots \ldots \ldots \ldots \ldots \ldots \ldots$

2.1 .1 Assembly Specification . . . . . . . . . . . . . . . . . . . . 4

2.1 .2 Fuel Pin and Guide Tube Specification. . . . . . . . . . . . . . . . . . 4

2.1 .3 Operational Specification $\ldots \ldots \ldots \ldots \ldots \ldots \ldots \ldots \ldots$

2.2 THE SCALE/DENOVO RADIATION TRANSPORT CODE $\ldots \ldots \ldots \ldots \ldots$

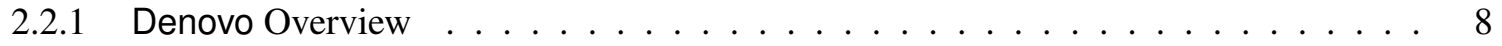

2.2 .2 Denovo Assembly Model $\ldots \ldots \ldots \ldots$. . . . . . . . . . . . 8

2.2 .3 Power from the Neutron Flux $\ldots \ldots \ldots \ldots$

2.3 THE THERMO-MECHANICS CAPABILITY WITHIN AMPFUEl . . . . . . . . . . 10

$2.3 .1 \quad$ Solver Strategy $\ldots \ldots \ldots \ldots \ldots \ldots \ldots$

$2.3 .2 \quad$ Heat Transfer within the Fuel, Gap, and Cladding . . . . . . . . . . . . . . . 11

2.3 .3 Mechanical Deformation of the Fuel and Cladding . . . . . . . . . . . . 13

2.3 .4 Geometric Simplifications $\ldots \ldots \ldots \ldots \ldots \ldots \ldots$

3 UNDERSTANDING SOLUTION TRANSFER AND MESH REFINEMENT CONSTRAINTS. . 15 3.1 SOLUTION TRANSFER FROM SMEARED CARTESIAN POWER TO RESOLVED UN-

STRUCTURED MESHES $\ldots \ldots \ldots \ldots \ldots \ldots \ldots \ldots \ldots \ldots \ldots$

3.2 EFFECT OF MESH REFINEMENT ON RUN-TIME . . . . . . . . . . . . . . . . 16

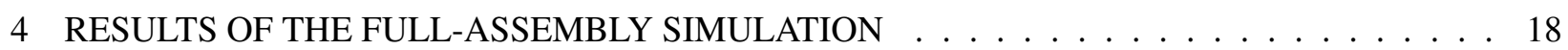

4.1 WORK FLOW AND COMPUTATIONAL REQUIREMENTS . . . . . . . . . . . . 18

4.2 NEUTRON FLUX $\ldots \ldots \ldots \ldots \ldots$

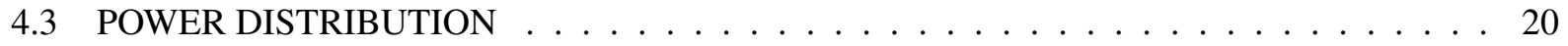

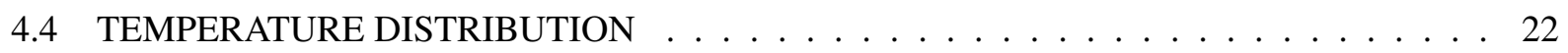

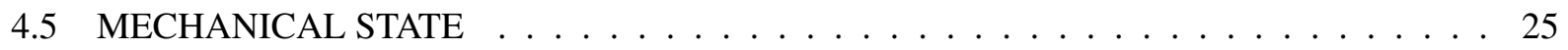

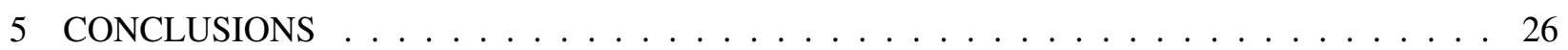

6 ACKNOWLEDGMENTS . . . . . . . . . . . . . . . . . . . . . . 32

REFERENCES . . . . . . . . . . . . . . . . . . . . . . . . . 34 



\section{List of Figures}

$1 \quad$ In-plane slice of the materials in the assembly. $\ldots \ldots \ldots \ldots \ldots$

$2 \quad$ Axial slice of the materials in the problem. $\ldots \ldots \ldots \ldots \ldots$

$3 \quad$ Denovo geometry with materials. . . . . . . . . . . . . . . . . . . . 9

$4 \quad$ Cross section of Denovo materials and coarse resolution mesh in fuel region. . . . . . . . . 9

$5 \quad$ Relative power distribution before and after smoothing from a coarse-mesh Denovo calcu-

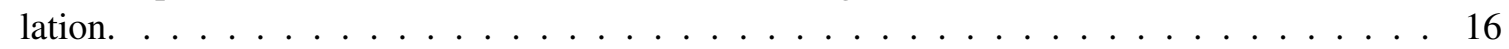

6 Relative power distribution from fine-mesh Denovo calculation and Zernike-smoothed coarse

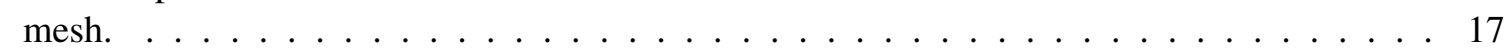

$7 \quad$ Denovo scalar neutron flux distribution at a fast energy. $\ldots \ldots \ldots \ldots$

$8 \quad$ Denovo scalar neutron flux distribution at a resonance energy. . . . . . . . . . . . . 21

9 Denovo scalar neutron flux distribution at a thermal energy. . . . . . . . . . . . . . 21

10 Power $\left(\frac{G W}{M T\left(\mathrm{UO}_{2}\right)}\right)$ distribution plotted on both the Denovo and AMPFuel meshes. $\ldots$. . . . 22

11 Power $\left(\frac{G W}{M T\left(U O_{2}\right)}\right)$ distribution scaled to clearly show the three-dimensional (axial, radial, and

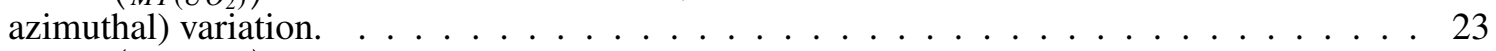

12 Power $\left(\frac{G W}{M T\left(\mathrm{UO}_{2}\right)}\right)$ distribution within the assembly 3 feet above the bottom of the active fuel

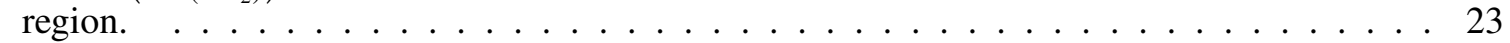

13 Power $\left(\frac{G W}{M T\left(U O_{2}\right)}\right)$ distribution within the assembly 5 feet above the bottom of the active fuel

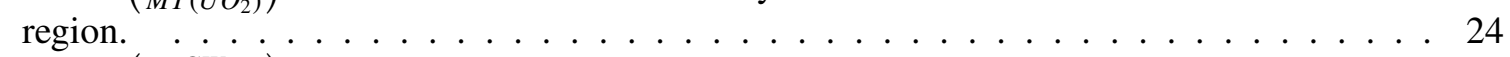

14 Power $\left(\frac{G W}{M T\left(U O_{2}\right)}\right)$ distribution within the assembly 7 feet above the bottom of the active fuel

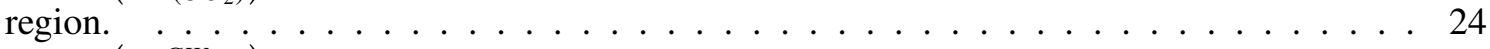

15 Power $\left(\frac{G W}{M T\left(U O_{2}\right)}\right)$ distribution within the assembly 9 feet above the bottom of the active fuel

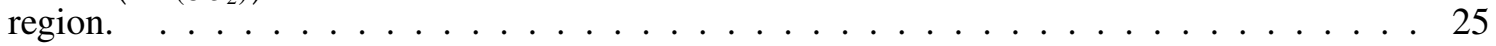

$16 \quad$ Temperature (Kelvin) distribution within the assembly. . . . . . . . . . . . . . 26

17 Temperature (Kelvin) distribution within the assembly for various heights above the bottom

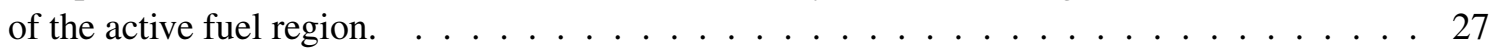

18 Total displacement magnitude (meters) on the initial AMPFuel mesh. . . . . . . . . . . . 28

19 Axial displacement (meters) on the initial AMPFuel mesh. . . . . . . . . . . . . . . 28

20 Total and axial displacement (meters) within the assembly 3 feet above the bottom of the active fuel region. $\ldots \ldots \ldots \ldots \ldots \ldots \ldots$

21 Total and axial displacement (meters) within the assembly 5 feet above the bottom of the

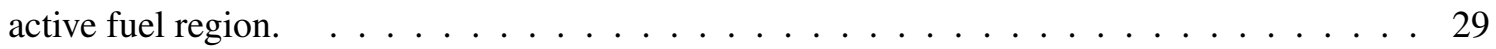

22 Total and axial displacement (meters) within the assembly 7 feet above the bottom of the

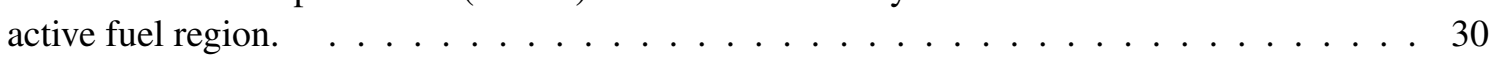

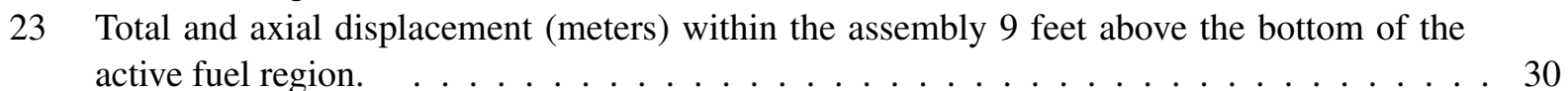





\section{List of Tables}

$1 \quad$ In-plane material definition of the fuel assembly $\ldots \ldots \ldots \ldots$

$2 \quad$ Axial description of the fuel assembly $\ldots \ldots \ldots \ldots \ldots \ldots$

$3 \quad$ In-plane description of the fuel pin and guide tube $\ldots \ldots \ldots \ldots \ldots$

$4 \quad$ Axial description of the fuel pin $\ldots \ldots \ldots \ldots \ldots \ldots$

$5 \quad$ Glossary of symbols in the gap conductance model $\ldots \ldots \ldots$. . . . . . . . . . . . . 14

$6 \quad$ Radiation transport spatial mesh and degrees of freedom counts $(\mathrm{M}=$ million $)$. . . . . . . 17

7 Thermo-mechanics element and degrees of freedom counts $(\mathrm{M}=$ million $) \ldots \ldots$. . . . . . . 18

$8 \quad$ Denovo and AMPFuel Run times for thermal problems $\ldots \ldots \ldots$. . . . . . . . 18 



\begin{abstract}
The Advanced Multi-Physics (AMP) Nuclear Fuel Performance code (AMPFuel) is focused on predicting the temperature and strain within a nuclear fuel assembly to evaluate the performance and safety of existing and advanced nuclear fuel bundles within existing and advanced nuclear reactors. AMPFuel was extended to include an integrated nuclear fuel assembly capability for (one-way) coupled radiation transport and nuclear fuel assembly thermo-mechanics. This capability is the initial step toward incorporating an improved predictive nuclear fuel assembly modeling capability to accurately account for source-terms and boundary conditions of traditional (single-pin) nuclear fuel performance simulation, such as the neutron flux distribution, coolant conditions, and assembly mechanical stresses.

A novel scheme is introduced for transferring the power distribution from the Scale/Denovo (Denovo) radiation transport code (structured, Cartesian mesh with smeared materials within each cell) to AMPFuel (unstructured, hexagonal mesh with a single material within each cell), allowing the use of a relatively coarse spatial mesh (10 million elements) for the radiation transport and a fine spatial mesh (3.3 billion elements) for thermo-mechanics with very little loss of accuracy. In addition, a new nuclear fuel-specific preconditioner was developed to account for the high aspect ratio of each fuel pin (12 feet axially, but $\frac{1}{4}$ inches in diameter) with many individual fuel regions (pellets).

With this novel capability, AMPFuel was used to model an entire $17 \times 17$ pressurized water reactor fuel assembly with many of the features resolved in three dimensions (for thermo-mechanics and/or neutronics), including the fuel, gap, and cladding of each of the 264 fuel pins; the 25 guide tubes; the top and bottom structural regions; and the upper and lower (neutron) reflector regions. The final, full assembly calculation was executed on Jaguar using 40,000 cores in under 10 hours to model over 162 billion degrees of freedom for 10 loading steps. The single radiation transport calculation required about $50 \%$ of the time required to solve the thermo-mechanics with a single loading step, which demonstrates that it is feasible to incorporate, in a single code, a high-fidelity radiation transport capability with a high-fidelity nuclear fuel thermo-mechanics capability and anticipate acceptable computational requirements.

The results of the full assembly simulation clearly show the axial, radial, and azimuthal variation of the neutron flux, power, temperature, and deformation of the assembly, highlighting behavior that is neglected in traditional axisymmetric fuel performance codes that do not account for assembly features, such as guide tubes and control rods.
\end{abstract}

\title{
1. INTRODUCTION
}

The ability to predict the behavior of nuclear fuel, at a continuum-scale, during irradiation in an operating reactor poses many significant challenges, including prediction of the effects of the microstructural evolution of the materials, the uncertainty and variability in the materials, and the impact of external source-terms and boundary conditions, with their uncertainty and variability. These external factors exist in the form of the power distribution, coolant conditions (temperature and pressure), mechanical stresses (debris, bowing, and fretting), and chemistry effects (oxidation and CRUD). This report documents the initial developments in the Advanced Multi-Physics (AMP) Nuclear Fuel Performance code (AMPFuel) [4] to incorporate an improved predictive nuclear fuel assembly modeling capability that accurately accounts for science domains that are beyond a single, isolated pin and traditionally modeled as source-terms or boundary conditions. Early work has focused on incorporating a physics-based detailed power distribution by leveraging the radiation transport capability from 
Scale/Denovo (Denovo) [6]. Previous studies have shown that uncertainty in the power distribution can account for significant uncertainty in the final fuel performance simulation [14].

This report describes an initial simulation to demonstrate the first step toward the integration of high-fidelity neutronics (capable of predictive neutron flux distributions) and fuel thermo-mechanics for assembly-level fuel performance analyses. Section 2 introduces the problem and software. Section 3 discusses challenges associated with providing computationally efficient solutions to such simulations. Section 4 details the results of the simulation, including the power distribution, thermo-mechanics, and computational assessment. The discussion of the problem is concluded in Section 5 .

This report satisfies the deliverable for the DOE Office of Nuclear Energy, Advanced Modeling and Simulation Office, milestone M2MS-12OR0602031, "Demonstrate AMP coupling to neutronics code on full LWR assembly," with the description: "AMP Nuclear Fuel Performance simulation that calls the Denovo radiation transport code to compute the flux distribution and then thermo-mechanics."

\subsection{TRADITIONAL NEUTRONICS SOURCE-TERM}

Traditional fuel performance codes, such as FRAPCON [3], model a single fuel pin in isolation and require the user to input the average pin power $\left(P_{\text {pin }}^{\tau}\right)$ and axial pin power distribution $\left(S_{\text {region }}^{\tau}\right)$ for a given time step $(\tau)$, in piece-wise constant "fuel regions," as a function of time (Eq. [1]) to provide a region-averaged power $\left(P_{\text {region }}\right)$ as a function of time $(t)$ :

$$
\begin{aligned}
P_{\text {region }}(t) & =P_{\text {pin }}^{\tau} S_{\text {region }}^{\tau} \\
\sum S_{\text {region }}^{\tau} H_{\text {region }} & =\sum H_{\text {region }}
\end{aligned}
$$

where Eq. [2] describes the axial normalization of the power distribution over the height $(H)$ of the pin. Within each "fuel region," effective (one energy-group) spatially-averaged neutron cross sections ( $\sigma_{x}^{i}$, where $i$ is an isotope of uranium or plutonium and $x$ is a reaction type) have been defined for the key transuranic isotopes along with a spatially flat (or radially varying) effective neutron flux $(\Phi)$ and radially varying effective uranium-238 (U238) capture cross section $\left[\sigma_{c}^{U 238}(r)\right.$ where $r$ is the radius of the fuel with a maximum of $R$ ] given by

$$
\sigma_{c}^{U 238}(r)=1+3.45 \exp \left[-3(R-r)^{0.45}\right] .
$$

These quantities are based on a series of MCNP simulations [10] with standard $\mathrm{UO}_{2}$ fuel in a PWR at a middle-of-life burnup. The fast neutron flux, which affects material models in the cladding through the fluence (time-integrated neutron flux), is specified by means of a user-defined magnitude or ratio of fast flux to average power [12,13,15]. This suite of approximations has been empirically and computationally tuned to provide sufficient accuracy for nominal operation with $\mathrm{UO}_{2}$ and $\mathrm{MOX}$ in well-defined problems, but it lacks the extensibility and generality required for predictive simulation. Significant limitations restricting the predictive nature of this approach include the inability to account for

- variations in the effective cross sections and flux distribution due to variations in isotopics, coolant, temperature, geometry, or materials;

- the azimuthally varying neutron flux distribution due to assembly features (such as control rods and neighboring pins or assemblies);

- the effect of within-region temperature distributions (in space and time) on effective cross sections and the neutron flux distribution; and 
- the inherent coupling of the physics (power, fuel temperature, gap thickness, and coolant temperature/density).

\subsection{POTENTIAL APPLICATIONS OF THE INTEGRATED CAPABILITY}

AMPFuel is being developed as a general toolset to improve predictions of the temperature and stresses in nuclear fuel assemblies; the key physical processes are comparable for a variety of reactor and fuel combinations. Therefore, this toolset can be applied to a variety of problems. This section describes two potential applications to which AMPFuel could be applied to gain additional insights. However, accurate modeling of these integrated systems will require additional research and development in coupling with thermal-fluid dynamics simulations.

\subsubsection{Fuel Failures in the Hatch Nuclear Power Plant}

The challenge of these multidimensional, multiphysics effects was exposed in a set of fuel failures in Unit 1 of the Hatch Nuclear Power Plant [2]. In Cycle 21, four fuel bundles had control blades inserted to Notch 14 for $2 \mathrm{GWd} / \mathrm{ST}$ (gigawatt-days per standard ton) at a burnup of $13.3 \mathrm{GWd} / \mathrm{ST}$. During a routine blade movement removing the control blades two notches, six fuel pins failed (due to pellet clad interaction) in four fuel bundles that were located in three out of four symmetric control cells. All of the failed pins were adjacent to the control blades.

When a control blade is removed, the space is filled with bypass water, significantly increasing the power level that previously was suppressed by the control rod. Many analyses have been performed to understand the cause of these failures, including the effects of local pin power peaking due to burnup gradients, fuel channel bowing, and transient thermo-mechanics [2]. Although the cause is still not fully-explained, simulations have shown that simplifications in the power distribution and irradiation history underpredicted the average and within-pin power distributions, and the azimuthally-symmetric fuel performance simulations were unable to properly account for the multidimensional thermo-mechanical response of the fuel pin as the power changed from peaking away from the control blade to peaking adjacent to the water channel.

\subsubsection{Shutdown Heat Removal Tests in the Experimental Breeder Reactor - II}

The Shutdown Heat Removal Tests (SHRT) demonstrated passive safety features of the EBR-II Experimental Breeder Reactor and included significant instrumentation. In collaboration with the Sharp team from Argonne National Laboratory, the AMPFuel will be integrated with the Nek computational fluid dynamics code to model the XX09 instrumented assembly in SHRT-17, a Loss of Flow with Scram experiment. This will provide a validation suite for high-fidelity assembly simulations that include within-assembly thermocouple (and flow meter) data for coolant temperatures at various elevations in the subassembly.

\section{BACKGROUND}

The development of an integrated neutronics and nuclear fuel performance capability for assembly-level simulations is relevant to many reactor and fuel concepts. For this report, the capability is 


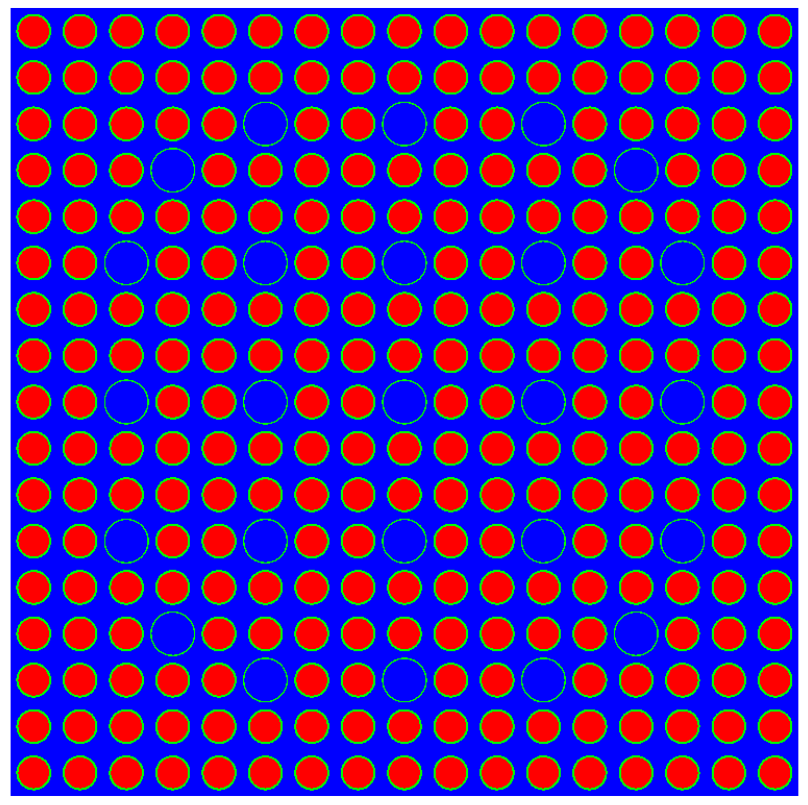

Fig. 1. In-plane slice of the materials in the assembly.

demonstrated on a full pressurized-water reactor (PWR) assembly using Denovo to provide a power distribution to the thermo-mechanics of AMPFuel.

\subsection{DEFINITION OF THE PROBLEM}

This demonstration problem is based on a $17 \times 17$ PWR that is from the "1_uox1.inp" from the MeshKit examples that Argonne National Laboratory has been developing and is similar to a $17 \times 17$ PWR that is of interest to the Consortium for Advanced Simulation of LWRs (light-water reactors), or CASL.

\subsubsection{Assembly Specification}

The array of fuel cells $(F C)$ and guide tube cells $(G T)$ in the assembly is defined with an assembly map shown in Table 1 and shown in Figure 1 . The cell pitch is 12.6 millimeters $(\mathrm{mm})$.

In this assembly model, the grid spacers will be neglected, but the effect of the coolant plenums (upper and lower) and assembly plates (bottom and top with handle) will be included, as shown in Figure 2 and defined in Table 2 .

\subsubsection{Fuel Pin and Guide Tube Specification}

Each fuel cell is composed of a single fuel pin surrounded by water coolant, as geometrically defined in Tables 3 and 4 . The fuel is composed of $5 \%$ enriched $\mathrm{UO}_{2}$ at $10.4 \mathrm{~g} / \mathrm{cc}$; the clad, plug, guide tube, and 
Table 1. In-plane material definition of the fuel assembly

\begin{tabular}{|c|c|c|c|c|c|c|c|c|c|c|c|c|c|c|c|c|}
\hline $\mathrm{C}$ & & & & & & & & & & & & & & & & \\
\hline $\mathrm{C}$ & & & & & & & & & & & & & & & & \\
\hline & & & & & & & & & & & & & & & C & $C$ \\
\hline & & & & & & & & & & & & & & & $\mathrm{C}$ & $\mathrm{FC}$ \\
\hline $\mathrm{C}$ & & & & & $\mathrm{FC}$ & & 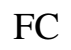 & & $\mathrm{FC}$ & & 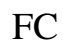 & & & & $\mathrm{C}$ & $C$ \\
\hline $\mathrm{C}$ & $\mathrm{C}$ & & C & & GT & & $\mathrm{C}$ & GT & $\mathrm{FC}$ & $\mathrm{FC}$ & GT & $\mathrm{FC}$ & C & GT & FC & $\mathrm{FC}$ \\
\hline $\mathrm{C}$ & & & $\mathrm{F}$ & & $\mathrm{F}$ & & & & & & $C$ & & & & C & $\mathrm{C}$ \\
\hline $\mathrm{C}$ & $\mathrm{C}$ & $\mathrm{F}$ & $\mathrm{C}$ & $\mathrm{FC}$ & $\mathrm{FC}$ & $\mathrm{FC}$ & $\mathrm{FC}$ & $\mathrm{FC}$ & $\mathrm{FC}$ & $\mathrm{FC}$ & FC & $\mathrm{FC}$ & $\mathrm{FC}$ & $\mathrm{FC}$ & $\mathrm{FC}$ & $\mathrm{FC}$ \\
\hline & & & & & $\mathrm{G}$ & & & $\mathrm{G}$ & $\mathrm{FC}$ & $\mathrm{F}$ & & $\mathrm{F}$ & & $\mathbf{J}$ & $\mathrm{C}$ & $C$ \\
\hline $\mathrm{C}$ & FC & $\mathrm{F}$ & FC & $\mathrm{F}$ & $\mathrm{FC}$ & $\mathrm{FC}$ & $\mathrm{C}$ & $\mathrm{FC}$ & $\mathrm{FC}$ & FC & $\mathrm{FC}$ & $\mathrm{F}$ & C & $\mathrm{GC}$ & FC & $\mathrm{FC}$ \\
\hline & & & & & $\mathrm{FC}$ & & & $\mathrm{F}$ & $\mathrm{FC}$ & $\mathrm{F}$ & $\mathrm{FC}$ & $\mathrm{F}$ & & $\mathrm{F}$ & $\mathrm{FC}$ & 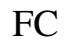 \\
\hline $\mathrm{FC}$ & $\mathrm{C}$ & $\mathrm{G}$ & $\mathrm{FC}$ & $\mathrm{F}$ & GT & $\mathrm{FC}$ & $\mathrm{C}$ & $\mathrm{G}^{\prime}$ & $\mathrm{FC}$ & $\mathrm{FC}$ & GT & FC & $\mathrm{C}$ & $\mathrm{GT}$ & $\mathrm{FC}$ & $\mathrm{FC}$ \\
\hline C & & & $\mathrm{FC}$ & & $\mathrm{FC}$ & & 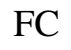 & $\mathrm{F}$ & $\mathrm{FC}$ & $\mathrm{F}$ & $\mathrm{FC}$ & $\mathrm{FC}$ & & $\mathrm{FC}$ & $\mathrm{FC}$ & $\mathrm{FC}$ \\
\hline FC & $\mathrm{FC}$ & & GT & $\mathrm{F}$ & $\mathrm{FC}$ & $\mathrm{F}$ & $\mathrm{FC}$ & $\mathrm{FC}$ & $\mathrm{FC}$ & $\mathrm{FC}$ & FC & $\mathrm{FC}$ & it & $\mathrm{FC}$ & $\mathrm{FC}$ & $\mathrm{FC}$ \\
\hline & $\mathrm{FC}$ & & $\mathrm{FC}$ & & GT & 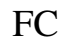 & & S & $\mathrm{FC}$ & $\mathrm{FC}$ & GT & $\mathrm{FC}$ & & $\mathrm{FC}$ & $\mathrm{FC}$ & $\mathrm{FC}$ \\
\hline $\mathrm{FC}$ & $\mathrm{FC}$ & $\mathrm{F}$ & $\mathrm{FC}$ & $\mathrm{F}$ & $\mathrm{FC}$ & FC & $\mathrm{FC}$ & $\mathrm{FC}$ & $\mathrm{FC}$ & $\mathrm{FC}$ & FC & $\mathrm{FC}$ & $\mathrm{FC}$ & $\mathrm{FC}$ & $\mathrm{FC}$ & $\mathrm{FC}$ \\
\hline FC & FC & 10 & FC & I & $\mathrm{FC}$ & FC & $\mathrm{CC}$ & $\mathrm{FC}$ & $\mathrm{FC}$ & $\mathrm{FC}$ & $\mathrm{F}$ & $\mathrm{FC}$ & 12 & $\mathrm{FC}$ & $\mathrm{FC}$ & $\mathrm{FC}$ \\
\hline
\end{tabular}

Table 2. Axial description of the fuel assembly

\begin{tabular}{|l|c|c|}
\hline Region & Height $(\mathrm{cm})$ & Height (in) \\
\hline Bottom reflector & 32.512 & 12.8 \\
Bottom plate & 15.240 & 6.0 \\
Fuel pin & 396.240 & 156.0 \\
Top plate & 15.240 & 6.0 \\
Upper reflector & 32.512 & 12.8 \\
Total height & 491.744 & 193.6 \\
\hline
\end{tabular}




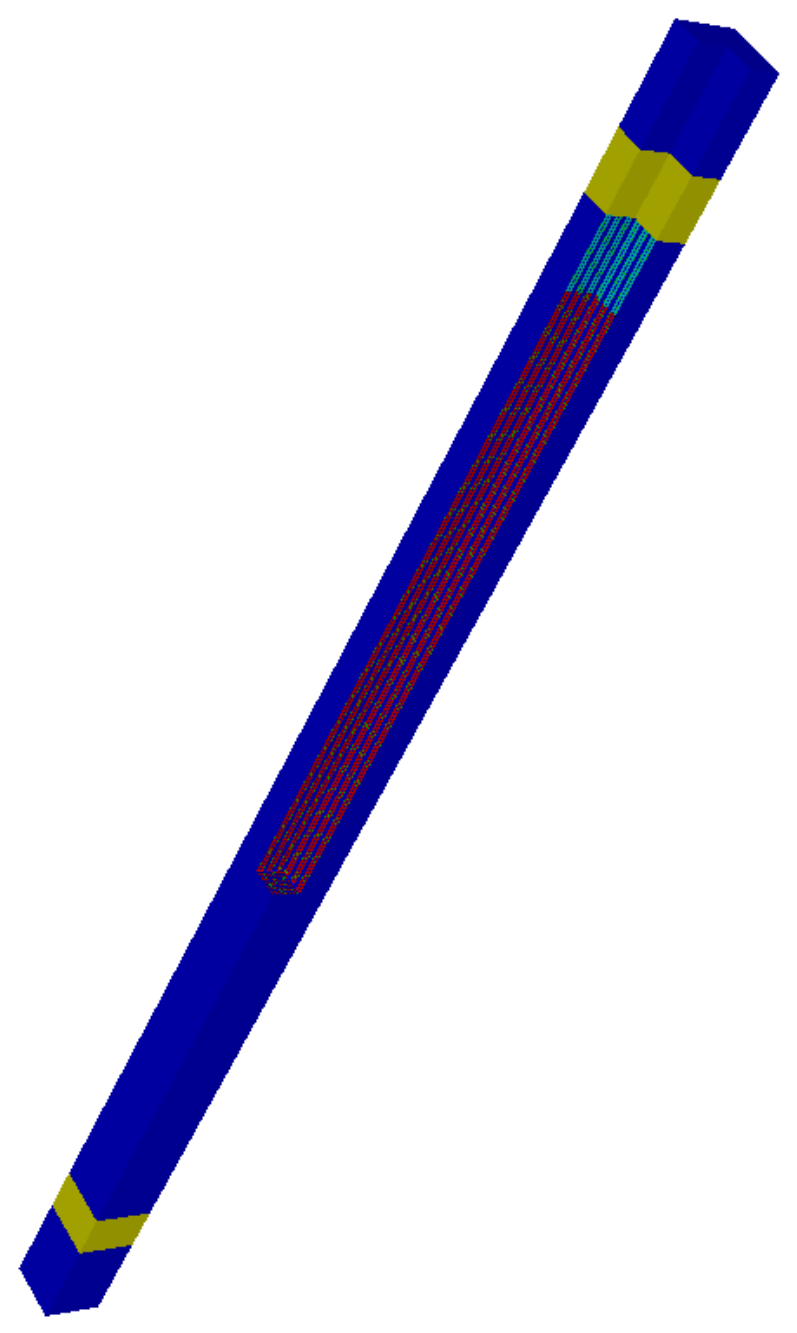

Fig. 2. Axial slice of the materials in the problem. 
top/bottom plate are composed of Zircaloy-4 at $6.55 \mathrm{~g} / \mathrm{cc}$; the gap is filled with pure helium at $0.0002 \mathrm{~g} / \mathrm{cc}$; and the coolant is $\mathrm{H}_{2} \mathrm{O}$ with $1000 \mathrm{ppm}$ natural boron.

Table 3. In-plane description of the fuel pin and guide tube

\begin{tabular}{|l|c|}
\hline Region & Radius or Pitch $(\mathrm{cm})$ \\
\hline Fuel & 0.4095 \\
Clad inner radius & 0.418 \\
Clad outer radius & 0.475 \\
Plug outer radius & 0.475 \\
Guide tube inner radius & 0.5715 \\
Guide tube outer radius & 0.6120 \\
Pincell width (pitch) & 1.26 \\
\hline
\end{tabular}

Table 4. Axial description of the fuel pin

\begin{tabular}{|l|c|c|}
\hline Region & Height (cm) & Height (in) \\
\hline Bottom plug & 1.016 & 0.4 \\
Single fuel pellet & 1.016 & 0.4 \\
Active fuel (360 pellets) & 365.76 & 144 \\
Upper plenum & 28.448 & 11.2 \\
Top plug & 1.016 & 0.4 \\
Total height & 396.24 & 156.0 \\
\hline
\end{tabular}

\subsubsection{Operational Specification}

The following problem specification was used to define the cold zero power (basis for thermal expansion) and hot full power.

- Cold Zero Power

- Temperature (of all materials) $=500 \mathrm{~K}$

- Power $=0 \mathrm{~W} / \mathrm{kg}\left(\mathrm{UO}_{2}\right)$

- Hot Full Power

- Coolant temperature $=555 \mathrm{~K}$

- Power $=20,000 \mathrm{~W} / \mathrm{kg}\left(\mathrm{UO}_{2}\right)=0.02$ giga-Watts per metric tonne of $\mathrm{UO}_{2}\left(\frac{G W}{M T\left(U O_{2}\right)}\right)$

- Burnup $=0$

\subsection{THE SCALE/DENOVO RADIATION TRANSPORT CODE}

Denovo is a framework for solving the Boltzmann radiation transport equation. It has the capability to solve the two-dimensional (2D) and three-dimensional (3D) deterministic multigroup discrete ordinates 
equations. A detailed description of the parallel algorithms and solvers can be found in References [5] and [6].

\subsubsection{Denovo Overview}

The deterministic component of Denovo makes use of the Koch-Baker-Alcouffe (KBA) [9] parallel spatial decomposition on nonuniform Cartesian grids using MPI-based communication. Volume-weighted subcell mixing of materials is used to offset geometric difficulties associated with the use of Cartesian meshes. The accuracy of Denovo has been verified for nuclear reactor simulation through the use of standard numerical benchmarks for radiation transport [16]. Additional parallelism in the form of an energy decomposition is also available, extending the parallel scalability to hundreds of thousands of processors [5,6]. Numerous spatial discretizations are available, as well as a variety of angular quadrature sets and boundary condition options. Advanced Krylov subspace solvers from several Trilinos [8] packages can be used to solve both the fixed-source and $k$-eigenvalue formulations of the transport equation with optional preconditioning in the form of diffusion synthetic acceleration (DSA) [11] and a novel multi-level energy preconditioner. High-performance parallel I/O for problem initialization and data analysis is available using HDF5 and SILO. The code system has been exercised heavily on OLCF (Oak Ridge Leadership Computing Facility) systems as part of the 2009 Petascale Early Science Project, the 2009 Visit Joule Project, and the 2010 Denovo Joule Project and as a 2010-2011 INCITE Project (NFI004).

\subsubsection{Denovo Assembly Model}

The geometry for the Denovo model is consistent with the prior description (with the axial distribution of regions described in Table 2) except that the fuel pin end plugs and fuel assembly end-plates are smeared with the coolant as a single material consisting of a mixture of $30 \%$ Zircaloy-4 and $70 \% \mathrm{H}_{2} \mathrm{O}$. The reflector regions are composed of $\mathrm{H}_{2} \mathrm{O}$ with $1000 \mathrm{ppm}$ natural boron. The Denovo model with materials is shown in Figure 3. Three different resolution Denovo models of the fuel assembly are used to evaluate the accuracy and computational cost of the transport simulation. The coarsest mesh consists of a $6 \times 6$ mesh in the $x-y$ plane for each pincell (a cell width of $2.1 \mathrm{~mm}$ ) combined with 121 cells in the axial direction (a cell height of $4.064 \mathrm{~cm}$ ) for a total of slightly over 1.25 million cells. The 2D layout of this mesh for a single axial region in the fuel (at the mid-plane) is shown in Figure 4. The middle-resolution mesh utilizes a $12 \times 12$ mesh per pincell in the $x-y$ plane (a cell width of $1.05 \mathrm{~mm}$ ) with 242 cells in the axial direction (a cell height of $2.032 \mathrm{~cm}$ ), for a total of slightly more than 10 million cells. The finest resolution Denovo model has a $24 \times 24$ resolution per pincell in the $x-y$ plane (a mesh width of $0.525 \mathrm{~mm}$ ) and the same axial resolution as the middle-resolution mesh, creating a problem with over 40 million cells. Results from the finest resolution mesh are not discussed in this report, but were used in testing and performance analysis.

Cross sections for the Denovo calculation were created using the Scale/CSASI sequence of the Scale [1] package using the 44-group ENDF/B-V (Evaluated Nuclear Data File) library. The fuel material consists of $5 \%$ enriched $\mathrm{UO}_{2}$ with a temperature that varies according to a cosine distribution along the axial length of the pin with a minimum temperature of $564 \mathrm{~K}$ and a maximum temperature of $900 \mathrm{~K}$. Temperatures are averaged along $12.192 \mathrm{~cm}$ axial regions providing 30 separate temperature regions. The moderator consists of $\mathrm{H}_{2} \mathrm{O}$ with $1000 \mathrm{ppm}$ natural boron at a temperature of $580 \mathrm{~K}$. The moderator density is varied linearly in the axial direction from a maximum of $0.745 \mathrm{~g} / \mathrm{cc}$ at the inlet to a minimum of 0.6656 $\mathrm{g} / \mathrm{cc}$ at the top of the fuel, with averaging taking place within the same 30 axial regions as used in the fuel processing.

A quadruple-range $(\mathrm{QR})$ angular quadrature is used for all calculations with four polar and four 


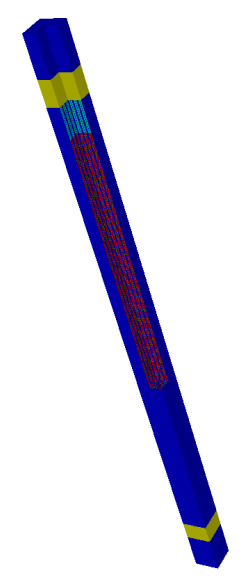

(a) Full Height

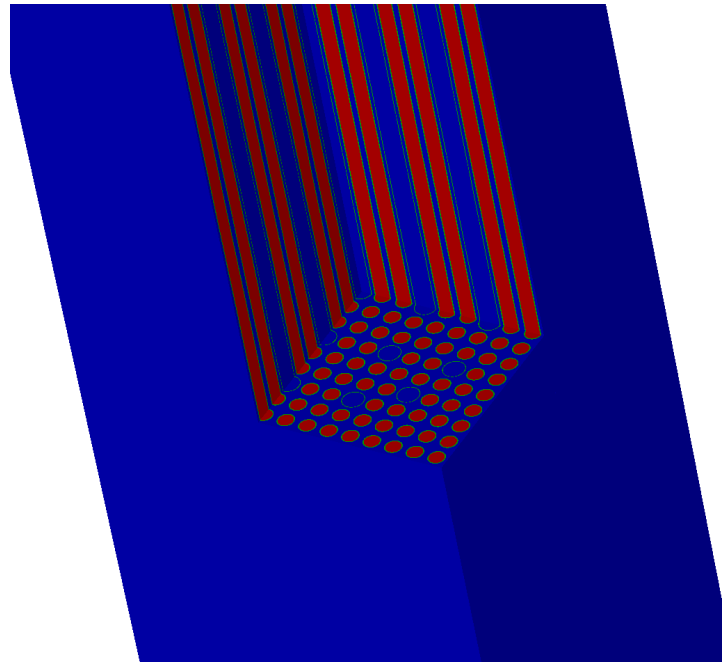

(b) Detail

Fig. 3. Denovo geometry with materials. Blue is moderator, red is fuel, green is clad, cyan is the plenum, and yellow is the smeared plug/plate.

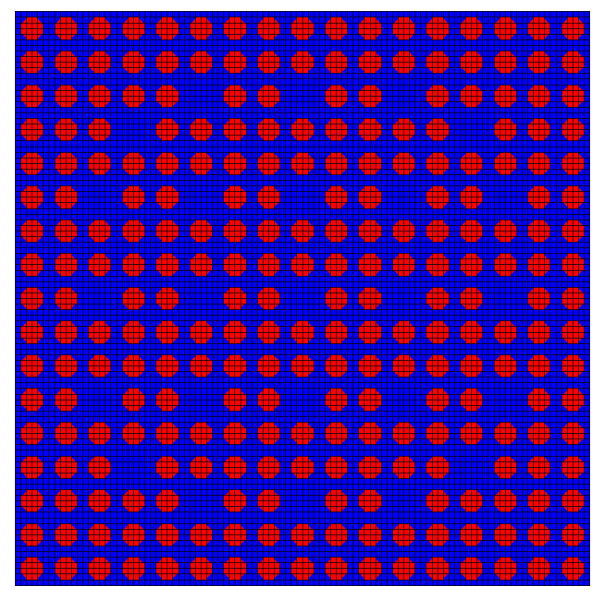

Fig. 4. Cross section of Denovo materials and coarse resolution mesh in fuel region. 
azimuthal levels per octant for a total of 128 angles. The total number of degrees of freedom (DOF; space, angle, and energy) for the Denovo problems ranges from around 7 billion for the coarsest mesh to nearly 227 billion for the finest problem. Denovo's Arnoldi eigensolver (which internally uses a multigroup GMRES [Generalized Minimal RESidual] algorithm for solving the relevant linear systems) is used for all solutions.

\subsubsection{Power from the Neutron Flux}

The specific power $\left(Q_{m}\right)$, which is the source term for the thermo-mechanics, is approximated as directly proportional to nuclear fission, thus neglecting gamma heating and neutron capture:

$$
\begin{aligned}
P & =\sum_{E=1}^{44} \Sigma_{f}^{E} \Phi^{E} \quad \text { in } \Omega, \\
Q_{m} & =P^{\prime}\left[\frac{Q_{u s e r} \rho d \Omega}{\int_{\Omega} P \rho d \Omega}\right] \text { in } \Omega,
\end{aligned}
$$

where $\Sigma_{f}^{E}$ is the macroscopic cross section for fission reactions in energy group $(E), \Phi^{E}$ is the neutron scalar flux, which is relative in magnitude, $P$ is the relative power based on the relative neutron flux, $\rho$ is the density of the fuel, $\Omega$ is the entire assembly spatial domain, $Q_{u s e r}$ is the user-defined magnitude of the average assembly specific power.

\subsection{THE THERMO-MECHANICS CAPABILITY WITHIN AMPFuel}

In normal operation, for nuclear fuel assemblies held together with a grid spacer (as opposed to wire wraps), the fuel pins are physically connected to the assembly structure only at grid-spacer springs and dimples, which (in normal operation) impose very little stress on the fuel pin. The fuel pins are set on top of the bottom assembly plate, with no weld to enforce contact, and have a "shoulder-height" separation from the bottom of the top assembly plate. Therefore, the fuel pins are isolated mechanically from one another and relatively isolated from the assembly structure.

The fuel pins are thermally coupled to other fuel pins only through the coolant conduction and convection, because there is negligible heat loss through the grid spacer. Because the flow is predominantly in the axial direction, there is relatively little heat transfer between "sub-channels" (regions between fuel pins) except near the grid-spacers and mixing vanes. Therefore, for this initial assembly simulation, the thermo-mechanics of the nuclear fuel pins are numerically approximated to be thermally and mechanically isolated.

\subsubsection{Solver Strategy}

For each fuel pin, the thermo-mechanics problem is solved by first solving the thermal problem in a parallel, fully coupled approach using a Jacobian-Free Newton-Krylov solver (using PETSc for the inexact Newton iteration and a flexible GMRES Krylov solver for the solution of linear systems). A similar approach is used for the mechanics with a multi-domain physics-based preconditioner designed specifically for nuclear fuel pins. The thermal preconditioner (using Trilinos for algebraic multi-grid) for the heat-transfer block is a parallel block-Jacobi (additive Schwarz) preconditioner in which each block involves inverting a thermal operator over a single volume (fuel region or cladding). The block Jacobi 
approach is a sufficiently strong preconditioner for this problem and allows all blocks to be inverted asynchronously. This implies that the time spent in preconditioning does not increase with the number of fuel regions. The preconditioner (using Trilinos for algebraic multi-grid) for the mechanics simulation is a physics-based extension of a parallel block-Gauss Seidel (multiplicative Schwarz) algorithm for the fuel region (where each block is represented as a single volume) that accounts for the upward movement of fuel regions due to the axial strain of the regions below. The clad mechanics preconditioner operates asynchronously with the preconditioner for the fuel regions. Here too a physics-based preconditioning approach has been developed which is highly asynchronous and shows promise.

Because AMPFuel provides the developer control over the strategies used to solve complex parallel problems, the thermo-mechanics portion of this problem can be formulated to have excellent parallel efficiency and scalability. The spatial domain is decomposed (in parallel) so that each fuel pin is on an independent set of processors, thus minimizing additional communication as the number of fuel pins increases. The result is an "embarrassingly parallel" (weak) scaling for the thermal calculation. For each fuel pin, the spatial domain of individual volumes (fuel regions and cladding) are decomposed so that each volume is on an independent set of processors. This ensures that no communication is required between volumes during the computationally expensive block-diagonal preconditioning of the heat transfer, which results in a very computationally efficient approach that will have excellent scalability as the number of fuel regions increases up to the number of pellets in the pin. Therefore, for a fuel assembly with hundreds of fuel pins (264), hundreds of fuel regions (360 pellets), and several coupled physics (2: heat transfer and mechanics), the problem could scale to a massive number of cores $(190,080)$ with absolutely no communication during the computationally expensive, multi-grid preconditioning. We note that our present strategy for mechanics preconditioning does involve communication between the various volumes, but the flexibility built into AMPFuel will allow us to continue experimenting rapidly with various forms of preconditioning. Even when the pins are modeled as physically coupled (through multidimensional flow modeling or fuel assembly distortion), we expect that the current approach will prove to be a highly efficient and scalable preconditioning strategy.

\subsubsection{Heat Transfer within the Fuel, Gap, and Cladding}

In this problem, the quasi-static conjugate heat transfer is modeled using nonlinear thermal conduction within each volume (fuel region and clad of each pin) and a Robin boundary condition to account for the transfer of heat between volumes (fuel-to-fuel and fuel-to-clad) and from the cladding to the coolant, described by the equations

$$
\begin{aligned}
& -\nabla \cdot[k \nabla T]=\rho Q_{m} \text { in } \Omega \\
& -k \nabla T+h T=h T^{\prime} \text { on } \partial \Omega \text {. }
\end{aligned}
$$

The coolant, like the gap, is not modeled as an explicit volume. In these equations $T$ is the temperature, $k$ is the thermal conductivity, $\rho$ is the density, $Q_{m}$ is the specific power, $h$ is the heat transfer coefficient of the gap or coolant, and $T^{\prime}$ is a projected map of the temperature of the surface of a neighboring volume $\left(d \Omega^{\prime}\right)$ onto the surface of the current volume $(\partial \Omega)$. The thermal conduction component of AMPFuel uses a standard continuous finite-element discretization of each volume. Specific power $\left(\frac{W}{k g\left(\mathrm{UO}_{2}\right)}\right)$ rather than power density $\left(\frac{W}{m^{3}}\right)$ is used as an input in AMPFuel; thus the power can easily be mapped from the Denovo solution to the thermo-mechanics mesh (even when the mesh is moving) because it is based on the initial geometry. 
The AMPFuel material coefficients for heat transfer $(k, \rho, h)$ from Eqs. (6) and (7) are based on FRAPCON [3]. The thermal conductivity $(k)$ of Zircaloy-4 depends on the temperature (T) according to the equation

$$
k[\mathrm{Zr}]=7.51+2.09 \times 10^{-2} T-1.45 \times 10^{-5} T^{2}+7.67 \times 10^{-9} T^{3} .
$$

The density ( $\rho$ ) of $\mathrm{UO}_{2}$ (Eq. 9), assuming the temperature is below the melting point (a valid assumption for this simulation), depends on the temperature (T) according to the relationship

$$
\rho\left[\mathrm{UO}_{2}\right]=10960\left(1.009-3 \times 10^{-5} T-0.12 e^{-5000 T^{-1}}\right)
$$

The thermal conductivity $(k)$ of $\mathrm{UO}_{2}(\mathrm{Eq} .10)$ is a function of the temperature $(\mathrm{T})$, burnup $(\mathrm{B})$, porosity $(\mathrm{P})$, and gadolinia content $(\mathrm{G})$ given by

$$
\begin{aligned}
& k\left[\mathrm{UO}_{2}\right]=\alpha\left(\left(\beta+\gamma T+\delta\left(1.0+\epsilon e^{-\frac{\zeta}{T}}\right)^{-1}\right)^{-1}+\eta T^{-2} e^{-\frac{\theta}{T}}\right), \\
& \text { where } \alpha=\frac{1.0789 P}{1+0.5(1-P)} \text {, } \\
& \beta=0.0452+1.1599 G+0.00187 B, \\
& \gamma=0.000246 \text {, } \\
& \delta=0.038\left(1.0-0.9 e^{-0.04 B}\right) B^{0.28}, \\
& \epsilon=396 \text {, } \\
& \zeta=6380 \text {, } \\
& \eta=3.5 \times 10^{9}, \\
& \text { and } \theta=16361 \text {. }
\end{aligned}
$$

Because there is no heat source in the cladding (gamma heating is neglected for now), the density of the zirconium is not required. The heat transfer coefficient $(h)$ accounts for conduction, convection, radiation, and discontinuities at materials interfaces, which is a function of contact pressure. The fuel-region-to-fuel-region $(f 2 f)$ and clad-to-water $(c 2 w)$ heat transfer coefficients take the fixed values

$$
\begin{aligned}
h[f 2 f] & =10 \\
\text { and } h[c 2 w] & =11,000 .
\end{aligned}
$$

The model that provides the fuel-to-clad $(f 2 c)$ heat transfer coefficient is applicable to both open and closed gaps and is nonlinearly dependent upon the gap thickness $\left(g_{f c}\right)$, temperature of the inner surface of the clad $\left(T_{c i}\right)$, and temperature of the outer surface of the fuel $\left(T_{f s}\right)$. The implementation is a reproduction 
of the original coding from FRAPCON, given by

$$
\begin{aligned}
& h[f 2 c]=h_{r}+h_{\text {gas }}+h_{\text {solid }}, \\
& \text { where } h_{r}=\sigma\left[T_{f s}^{2}+T_{c i}^{2}\right]\left[T_{f s}+T_{c i}\right] /\left[1 / e_{f}+\left(r_{f s} / r_{c i}\right)\left(1-1 / e_{c}\right)\right] \text {, } \\
& h_{\text {gas }}=k_{\text {gas }} /\left(d_{\text {eff }}+1.8 g_{f c}-b-d\right) \text {, } \\
& h_{\text {solid }}=\frac{5 K_{m}}{R E}\left\{\begin{array}{lr}
P_{\text {rel }} R_{\text {mult }}, & \text { if } P_{\text {rel }}>0.003 \\
.003, & \text { if } 0.03>P_{\text {rel }}>9.010^{-6} \\
\sqrt{P_{\text {rel }}} & \text { if } P_{\text {rel }}<9.010^{-6}
\end{array},\right. \\
& d_{\text {eff }}=\left\{\begin{array}{l}
\exp (-P / 800)\left(R_{f}+R_{c}\right), \quad \text { closed } \\
R_{f}+R_{c}, \quad \text { open }
\end{array}\right. \\
& g_{f c}=\left(A k_{\text {gas }}^{\sqrt{T_{\text {gas }}}} / P_{\text {gas }}\right) / \sum_{i} \frac{a_{i} f_{i}}{\sqrt{M_{i}}} \\
& \sigma=5.6697 \mathrm{H} \times 10^{-8}\left(\mathrm{~W} / \mathrm{m}^{2}\right) \mathrm{K}^{4}, \\
& b=1.397 \mathrm{H} \times 10^{-6} \mathrm{~m}, \\
& A=0.7816 \text {, } \\
& K_{m}=2 K_{f} K_{c} /\left(K_{f}+K_{c}\right) \text {, } \\
& R=\sqrt{R_{f}^{2}+R_{c}^{2}} \\
& R_{\text {mult }}=\left\{\begin{array}{r}
\frac{1000}{3 P_{\text {rel }}}, \text { if } P_{\text {rel }} \leq 0.0087 \\
2.9, \text { if } P_{\text {rel }}>0.0087
\end{array}\right. \text {, } \\
& E=\exp \left[5.738-0.528 \ln R_{2}\right] \text {. }
\end{aligned}
$$

The gap thickness $\left(g_{f c}\right)$ at a given location is computed as the radial projection from the current node to the surface of the adjacent volume (fuel or clad). Table 5 provides an interpretation of the symbols, and associated units, used in the gap conductance model. The quantities $e_{f}, e_{c}, k_{g a s}, K_{f}, K_{c}, a_{i}, P_{\text {gas }}$ have further mathematical models associated with them which are provided through the FRAPCON routines and documented in the FRAPCON manual. Note that the FRAPCON manual does not describe the parameter $H$.

The gap conductance model was used in many of the simulations within this report. The final simulation, however, used a constant value of 9650 .

\subsubsection{Mechanical Deformation of the Fuel and Cladding}

The mechanics component of AMPFuel approximates the governing equation that describes a body, $\Omega$,

undergoing an infinitesimal strain and displacement. At static mechanical equilibrium the body satisfies the condition

$$
\nabla \sigma+\rho b=0 \quad \text { in } \quad \Omega .
$$


Table 5. Glossary of symbols in the gap conductance model

\begin{tabular}{|l|l|c|}
\hline Symbol & Name & $\mathrm{Units}$ \\
\hline$\rho$ & Density & $\mathrm{kg} \mathrm{m}^{-3}$ \\
$T$ & Temperature & $\mathrm{K}$ \\
$k$ & Thermal conductivity & $\mathrm{m}^{-1} \mathrm{~K}^{-1}$ \\
$Q$ & Heat source or reactor power & $\mathrm{Wm}^{-3}$ \\
$q$ & Heat flux & $\mathrm{Wm}^{-2}$ \\
$C_{p}$ & Specific heat at constant pressure & $\mathrm{kg}^{-1} \mathrm{~K}^{-1}$ \\
$r$ & Spatial position vector & $\mathrm{m}$ \\
$v$ & Surface normal & \\
$\sigma$ & Sefan-Boltzmann constant & $\mathrm{K}^{4} \mathrm{~W} / \mathrm{m}^{2}$ \\
$T_{f s}$ & Fuel outer surface temperature & $\mathrm{K}$ \\
$T_{c i}$ & Clad inner surface temperature & $\mathrm{K}$ \\
$e_{f}$ & Fuel emissivity & \\
$e_{c}$ & Cladding emissivity & \\
$r_{f s}$ & Fuel outer surface radius & $\mathrm{m}$ \\
$r_{c i}$ & Cladding inner surface radius & $\mathrm{m}$ \\
$k_{g a s}$ & Gas thermal conductivity & $\mathrm{KW} / \mathrm{m}$ \\
$d$ & Fuel-cladding gap size & $\mathrm{m}$ \\
$P_{r e l}$ & Interfacial pressure to cladding Meyer hardness & \\
$P$ & Fuel-cladding interfacial pressure & $\mathrm{kg} / \mathrm{cm}$ \\
$R_{f}$ & Fuel roughness & \\
$R_{c}$ & Clad roughness & $\mathrm{K}$ \\
$T_{\text {gas }}$ & Gas temperature & $\mathrm{Pa}$ \\
$P_{g a s}$ & Gas pressure & $\mathrm{KW} / \mathrm{m}$ \\
$K_{f}$ & Fuel thermal conductivity & \\
$K_{c}$ & Clad thermal conductivity & \\
$a_{i}$ & Accomodation coefficient i-th gas component & \\
$f_{i}$ & Mole fracion of i-th component & \\
$M_{i}$ & Gram-molecular weight of i-th gas component & $\mathrm{kmoles}$ \\
$R_{2}$ & Roughness of the rougher surface & \\
\hline & & \\
\hline
\end{tabular}

Here, $\sigma, \rho$, and $b$ are the symmetric Cauchy stress tensor, density, and body forces, respectively. $\sigma$ is a function of the displacements, $u$; the precise form of this function depends on the material used in the body. This is solved with a standard finite-element methodology.

The mechanics material models provide the correct estimate of stress $\left(\sigma_{i j}\right)$ at each Gauss point subjected to some external strain $\left(\epsilon_{i j}\right)$, based on the deformation mechanisms (e.g. thermal expansion, swelling, densification) causing the displacement of the fuel pellet and the clad. These material models also return the constitutive matrix $\left(C_{i j k l}\right)$ at each Gauss point, which relates the strain with the stress:

$$
\sigma_{i j}=C_{i j k l} \epsilon_{k l} \text {. }
$$

Because this problem is based on fresh fuel, small strain conditions are assumed and the densification and swelling are included, but negligible. Thus the total strain $\left(\epsilon_{i j}^{t o t}\right)$ can be written in additive form:

$$
\epsilon_{i j}^{t o t}=\epsilon_{i j}^{e}+\epsilon_{i j}^{p}+\epsilon_{i j}^{t h}+\epsilon_{i j}^{s w}+\epsilon_{i j}^{d e}+\epsilon_{i j}^{c}+\epsilon_{i j}^{r e},
$$


where $\epsilon_{i j}^{e}$ is the elastic strain; $\epsilon_{i j}^{p}$ is the plastic strain; $\epsilon_{i j}^{t h}$ is the strain due to thermal expansion; $\epsilon_{i j}^{s w}$ is the swelling strain; $\epsilon_{i j}^{d e}$ is the densification strain; $\epsilon_{i j}^{c}$ denotes the strain due to temperature, irradiation and stress-induced creep; $\epsilon_{i j}^{r e}$ signifies the relocation strain. All strains are computed implicitly, and most properties are a function of temperature.

\subsubsection{Geometric Simplifications}

Rather than model every fuel pellet explicitly, the fuel was decomposed into effective "fuel regions." In a fine-mesh simulation, every pellet could be resolved, but it has not been determined that this is required for understanding the assembly performance. Therefore, in the initial implementation, the fuel pellets will be smeared into a series of effective fuel regions (from four pellets smeared to a smearing of the entire fuel pin). The presence of the plugs, top and bottom plates, guide tubes, and within-pin springs will be neglected.

\section{UNDERSTANDING SOLUTION TRANSFER AND MESH REFINEMENT CONSTRAINTS}

In this section, we present two aspects that were required to properly understand and evaluate the capability and performance of the code for assembly-level simulations. The solution transfer between codes is described in Section 3.1 and the effect of mesh refinement on run-time in Section 3.2.

\subsection{SOLUTION TRANSFER FROM SMEARED CARTESIAN POWER TO RESOLVED UNSTRUCTURED MESHES}

Denovo computes a flux (power) distribution on a Cartesian mesh that will usually be significantly coarser than the mesh used by AMPFuel for thermo-mechanics calculations. Directly mapping coarse Denovo powers onto a fine AMPFuel mesh may result in a "staircase" effect by which the coarse power distribution produces highly nonphysical asymmetries in the temperature and mechanics solutions.

To counteract such behavior, a smoothing operation has been introduced in the form of a polynomial expansion of the power within each fuel pin. In particular, we make use of the Zernike polynomials in the $x-y$ plane and the Legendre polynomials in $z$. The Zernike polynomials form a complete set of orthogonal functions on the unit disk and are denoted $Z_{n}^{m}(x, y)$, where $n$ is the degree and $m$ is the order of the polynomial. The Legendre polynomials form a complete set of orthogonal polynomials on a line segment and are denoted $P_{\ell}(z)$, where $\ell$ is the degree of the polynomial. Combining these polynomials gives a complete expansion for a function on a cylinder, i.e.,

$$
F(x, y, z)=\sum_{\ell=0}^{L} \sum_{n=0}^{N} \sum_{m=-n}^{n} C_{n, m}^{\ell} Z_{n}^{m}(x, y) P_{\ell}(z),
$$

where $C_{n, m}^{\ell}$ is an expansion coefficient, $L$ is the prescribed degree of the Legendre expansion, and $N$ is the degree of the Zernike expansion. Evaluating these coefficients is accomplished by exploiting the orthogonality of the polynomials, producing the expression

$$
C_{n, m}^{\ell}=\frac{(n+1)(2 \ell+1)}{\left(1+\delta_{m, 0}\right) \pi} \iint_{x^{2}+y^{2} \leq 1} d x d y \int_{-1}^{1} d z F(x, y, z) Z_{n}^{m}(x, y) P_{\ell}(z)
$$




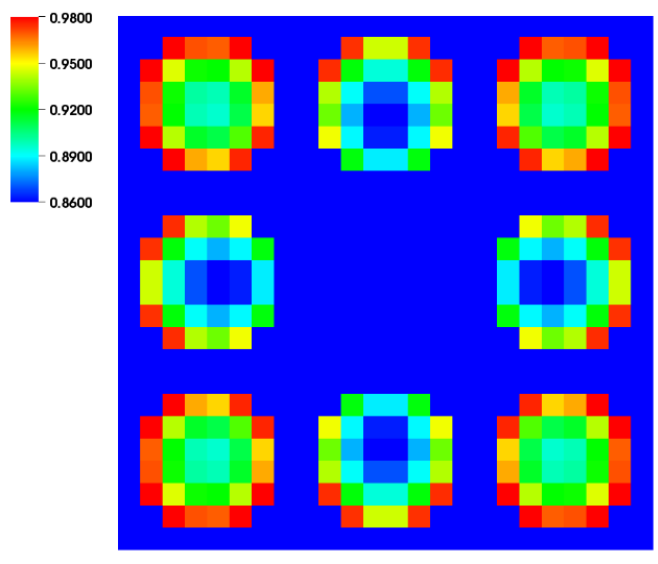

(a) Coarse-Mesh Denovo Power

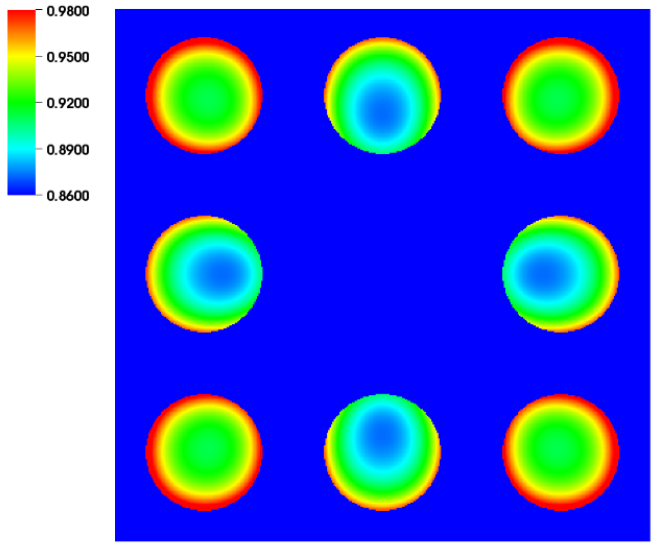

(b) Zernike-Smoothed Power from Coarse-Mesh

Fig. 5. Relative power distribution before and after smoothing from a coarse-mesh Denovo calculation.

where $\delta_{n, m}$ is the Kronecker delta function. Once these coefficients have been evaluated for a particular flux or power distribution on the Denovo mesh (a separate set of coefficients will be computed for each region of each pin), Eq. (17) can be used to map the distribution onto the desired locations on the AMPFuel mesh. To demonstrate the smoothing property of the Zernike polynomials, we consider a 2D $3 \times 3$ "mini-assembly" consisting of eight $\mathrm{UO}_{2}$ fuel rods surrounding a $\mathrm{B}_{4} \mathrm{C}$ control rod. The power distribution is first computed on a coarse mesh (a $24 \times 24$ mesh over the entire domain) using the $2 \mathrm{D}$ step characteristics spatial discretization in Denovo, producing the profile shown in Figure 5a, with units that are unnormalized. From this expansion the coefficients of Eq. (18) (neglecting the $z$ component because this is a 2D calculation) are computed, and Eq. (17) is used to map the expansion onto a much finer $384 \times 384$ mesh with the result shown in Figure 5 b. The result is a dramatically smoother distribution, and in excellent agreement with the result of a complete Denovo calculation performed on the fine mesh, shown in Figure 6a. The fine mesh calculation required approximately 15 minutes to complete, whereas the coarse mesh calculation in addition to the mapping onto the fine mesh required only four seconds [7].

Note that the "coarse mesh" in this study (an $8 \times 8$ mesh per pincell) is between the coarse mesh $(6 \times 6)$ and the medium mesh $(12 \times 12)$ of the full assembly simulations. All meshes used in the 3D calculations are significantly coarser than the "fine mesh" $(128 \times 128)$ in this study. Therefore, in the full assembly, the the medium Denovo mesh mapped onto the thermo-mechanics mesh should be sufficiently accurate to replicate the solution of a much finer Denovo mesh and still provide a smooth source-term for the thermo-mechanics.

\subsection{EFFECT OF MESH REFINEMENT ON RUN-TIME}

An important aspect of any large-scale parallel computation is that the performance scale well when the number of processors being used increases. In this section, we look at the results of two different resolution calculations for coupled radiation transport and heat transfer. The Denovo models are the same as the 


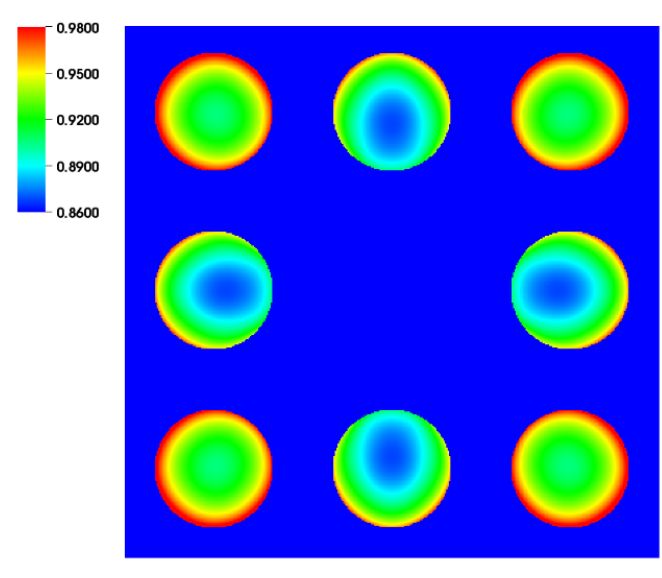

(a) Fine-Mesh Denovo Power

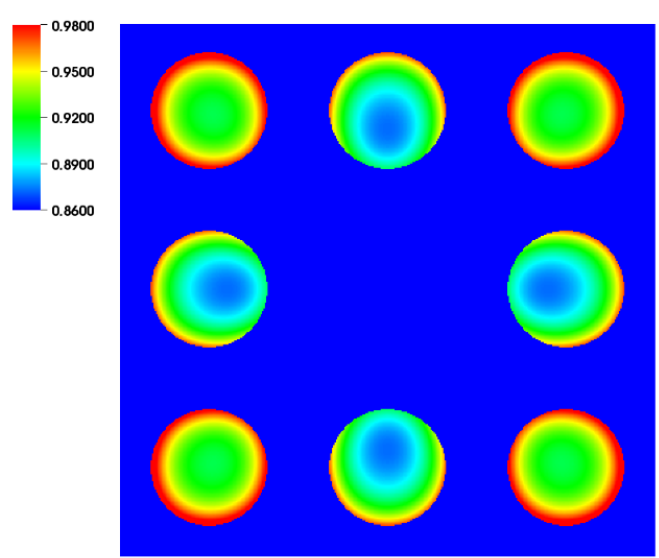

(b) Zernike-Smoothed Power from Coarse-Mesh

Fig. 6. Relative power distribution from fine-mesh Denovo calculation and Zernike-smoothed coarse mesh.

coarse and middle-resolution models described in Section 2.2.2, the problem sizes are shown in Table 6 . The cell counts are the global number of spatial cells, and the total DOF is the cell count multiplied by the number of angles (128) and the number of energy groups (44). It should be noted that because Denovo is solving an integral form of the radiation transport equation, the angular components do not appear in the solution vector and thus the size of the vector that must be stored is smaller than the reported DOF count. However, because every iteration requires treating space, energy, and angle, the DOF count is a more reliable indicator of the computational effort required for a calculation. Two different mesh resolutions (Mesh 1 and Mesh 2) were used for the thermal simulations in this study; the size of these meshes is given in Table 7(Mesh 3 was only used in the full assembly simulation discussed in Section 4). The number of DOF in the thermal calculation is the number of elements multiplied by eight Gauss points per element; thermo-mechanics requires 3 additional DOFs per gauss point.

Table 6. Radiation transport spatial mesh and degrees of freedom counts (M=million)

\begin{tabular}{|c|c|c|}
\hline & Mesh 1 & Mesh 2 \\
\hline Spatial cells & $1.3 \mathrm{M}$ & $10 \mathrm{M}$ \\
Energy groups & 44 & 44 \\
Discrete ordinates & 128 & 128 \\
Total DOF & $7,100 \mathrm{M}$ & $57,000 \mathrm{M}$ \\
\hline
\end{tabular}

Two calculations were performed, one using the coarse Denovo mesh as well as the coarse AMPFuel mesh on 578 processors, and the other using both fine meshes on 4624 processors. The run times for each component of the computation are shown in Table 8 . Because the size of the Denovo problem and the number of processors both increase by a factor of eight, this represents a weak scaling study for Denovo and the run time should (ideally) show little growth. Although the Denovo run time went up significantly, 
Table 7. Thermo-mechanics element and degrees of freedom counts ( $M=$ million)

\begin{tabular}{|c|c|c|c|}
\hline & Mesh 1 & Mesh 2 & Mesh 3 \\
\hline Clad elements & $55 \mathrm{M}$ & $55 \mathrm{M}$ & $55 \mathrm{M}$ \\
Pellet elements & $60 \mathrm{M}$ & $444 \mathrm{M}$ & $3,250 \mathrm{M}$ \\
Total elements & $115 \mathrm{M}$ & $499 \mathrm{M}$ & $3,300 \mathrm{M}$ \\
Total thermal DOF & $918 \mathrm{M}$ & $4,000 \mathrm{M}$ & $26,000 \mathrm{M}$ \\
Total thermo-mechanics DOF & $918 \mathrm{M}$ & $16,000 \mathrm{M}$ & $105,000 \mathrm{M}$ \\
\hline
\end{tabular}

Table 8. Denovo and AMPFuel Run times for thermal problems

\begin{tabular}{|c|c|c|c|c|}
\hline & \multicolumn{2}{|c|}{ Mesh 1 } & \multicolumn{2}{c|}{ Mesh 2 } \\
\hline & Wall time (min) & CPU hours & Wall time (min) & CPU hours \\
\hline Denovo time & 23.9 & 230 & 65.2 & 5024 \\
AMP time & 24.5 & 236 & 21.0 & 1618 \\
Total time & 48.4 & 466 & 86.2 & 6643 \\
\hline
\end{tabular}

the increase is largely explained by a significant increase in the number of iterations required (from 15 to 27), possibly due to inadequate selection of subspace dimensions for the larger problem. It does not indicate a lack of scalability on the part of Denovo, because it has been shown to scale well on similar problems in previous studies [5]. The time required to complete the AMPFuel portion of the calculation actually decreased from the coarse mesh to the fine mesh. The decrease is partially due to the fact that the number of DOF in the AMPFuel problem increased by slightly more than a factor of four, whereas the number of processors increased by a factor of eight, resulting in less work per processor for the fine-mesh case. For this particular problem, there was no optimization and the scaling study was non-ideal because the cladding mesh remained constant (55 million elements) and the fuel pellet meshes were each refined (from 60 million to 444 million elements). This produced an inconsistency in the true scaling study (for the cladding it was strong, for the fuel it was weak). In addition, this is not simply solution time, but total execution time, which includes the startup and file $\mathrm{I} / \mathrm{O}$.

\section{RESULTS OF THE FULL-ASSEMBLY SIMULATION}

The final full-assembly simulation was completed on Monday, January 16, after 10 hours on 40,000 cores of the Jaguar (Cray XT5) supercomputer at the OLCF. This section details the results of that simulation, including a description of the computational requirements and timing in Section 4.1, the multi-group neutron flux in Section 4.2, the power distribution both computed with Denovo and mapped onto the AMPFuel mesh in Section 4.3, the temperature distribution within the cladding and fuel pins in Section 4.4, and the displacement within the fuel and cladding due to thermal expansion in Section 4.5 .

\subsection{WORK FLOW AND COMPUTATIONAL REQUIREMENTS}

Problem setup and mesh generation for AMPFuel and Denovo were performed independently, using their standard automated mesh generation capabilities. The mesh generation for the full-assembly Denovo simulation completed in several seconds, whereas AMPFuel required approximately 4 minutes to generate the cladding mesh and several seconds for the fuel region. The input files and meshes were copied to the 
Jaguar (Cray XT5) supercomputer for execution. At the time, Jaguar had no wait-time in the queue for this job, and the total simulation required 10 hours on 40,000 cores. Therefore, the total workflow time was under 11 hours to generate the meshes and execute the problem.

The final simulation included the "Mesh 2" from Denovo and the "Mesh 3" from thermo-mechanics, as defined in Tables 6 and 7, respectively. This produced an integrated problem with a total of 162 billion DOF. In the final simulation, the timing routines were errantly removed, so the following execution times are approximate, based on experience.

Of the 10 hours of wall-clock time, the execution time was approximately:

- AMPFuel initialization: 2 minutes

- Denovo initialization: 10 seconds

- Denovo execution: 55 minutes

- Solution transfer to AMPFuel mesh: 30 seconds

- AMPFuel problem setup: 4 minutes

- AMPFuel thermo-mechanics (10 loading steps): 9 hours

- AMPFuel single thermal time step: 10 minutes

- AMPFuel single mechanics loading time step:

* AMPFuel minimum: 10 minutes

* AMPFuel average: 45 minutes

* AMPFuel maximum: 75 minutes

\subsection{NEUTRON FLUX}

The (unnormalized) scalar neutron flux distribution computed by Denovo is shown in Figures $7-9$ for three different energy groups, where the aspect ratio (height to width) is scaled by a factor of 10 in the (b) plots to more easily see the within-assembly variations. Although the Denovo calculation included 44 energy groups, we present these three groups as representative of a typical fast (group 5; $2.5-3.0$ million electron volts $[\mathrm{eV}]$ ), resonance (group 20; 6-8.1 eV), and thermal (group 38; 0.04-0.05 eV) energy group. The scalar neutron flux $\left(\frac{\text { neutrons }}{\mathrm{cm}^{2}-\mathrm{sec}}\right)$ that arises from Denovo, or any k-eigenvalue radiation transport code, is a relative quantity; in these plots the magnitude has not been scaled to produce the integrated user-defined specific power $\left(20 \mathrm{~kW} / \mathrm{kg}\left[\mathrm{UO}_{2}\right]\right)$ but represents the relative values as a function of space within an energy group and between energy groups.

These groups illustrate the general trends that should be expected in a light-water reactor assembly. In particular, the thermal group (Figure 9) clearly displays sharp gradients between the guide tubes and the fuel pins, which are significant because they lead to corresponding variations in the power distribution that can be captured only in a 3D coupled simulation. The fast group (Figure 7) solution shows that there is a significant spatial distribution to the fast flux, which is the driver for clad growth and some forms of fuel assembly distortion. The resonance group (Figure 8) solution demonstrates that Denovo, even with a relatively coarse mesh, can capture the very strong radial neutron flux distribution that leads (through neutron capture of uranium-238) to the production of plutonium-239 in the outer rim of the fuel and a high-power "rim effect" in high-burnup fuel. This "rim effect" is modeled in most legacy fuel performance codes but often neglected in traditional reactor physics simulations, which are generally not focused on the 


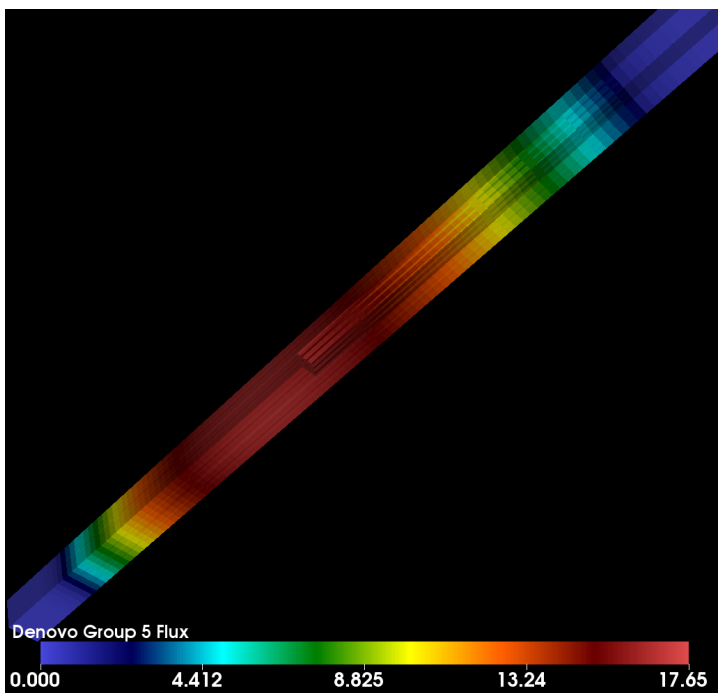

(a) No Scaling

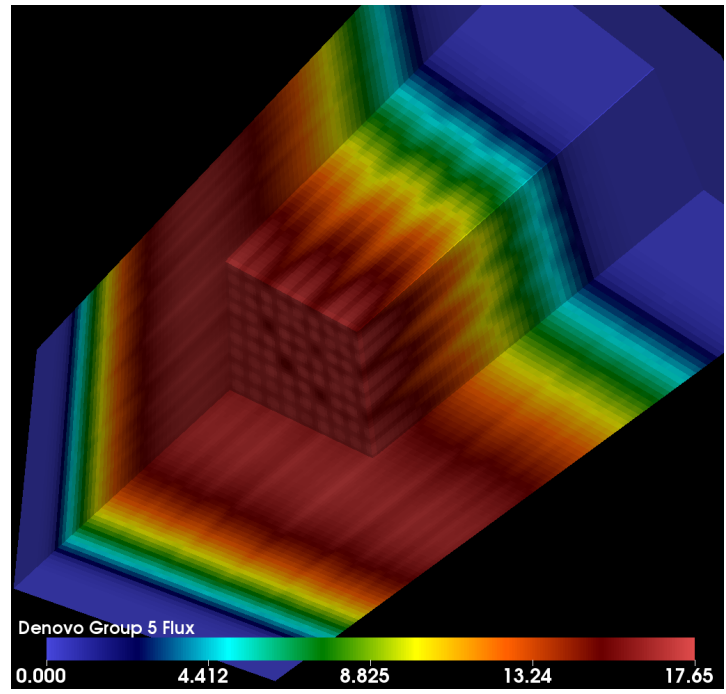

(b) Scaled (10x reduction in height)

Fig. 7. Denovo scalar neutron flux distribution at a fast energy.

within-pin power distribution. Therefore, additional studies will be required to evaluate the sensitivity of the power distribution during depletion as the Denovo mesh is refined and if the Zernike mapping of the neutron flux in each energy group can accurately capture that steep gradient; it is possible that an unstructured mesh radiation transport code, such as PROTEUS, from Argonne National Laboratory, may prove more efficient for this particular aspect of the problem.

Although these trends are expected in a reactor physics simulation, this fidelity of neutron flux distribution has never been available within a nuclear fuel performance code to explore the impact of "traditional" approximations (e.g., axisymmetric power distribution, axially-constant power in a small set of axial regions). Also, this capability will allow early users to understand the impact of assembly features that may prove significant for a set of problems but that were neglected in this initial demonstration, such as grid spacers, control rods, and neighboring assemblies. In addition, this capability will allow users to model different reactor/fuel types (e.g., mixed-oxide fuel, boiling-water reactors, advanced fuels/reactors) with relative simplicity and high accuracy.

\subsection{POWER DISTRIBUTION}

The power distribution is derived from the multi-group neutron flux, as described in Section 2.2.3, and shown in $\frac{G W}{M T\left(U O_{2}\right)}$ for the full assembly in both Denovo and AMPFuel (Figure 10. Because of the high aspect ratio of the problem, the vertical axis has been reduced by a factor of 10 and displayed in Figure 11 . along with a higher minimum value, to more easily see the multidimensional aspect of the power distribution.

Figure 11a, from Denovo, displays the zero power regions in the cladding and coolant as blue, whereas Figure $11 \mathrm{~b}$, from the thermo-mechanics, shows the cladding as zero power but does not display the coolant or plenum regions because they were not part of the thermo-mechanics model (mesh or solution). The axial variation is peaked below the mid-plane because of additional moderation owing to the higher density of the colder coolant in the lower region. The axial power shape is relatively similar from pin to pin, but not 


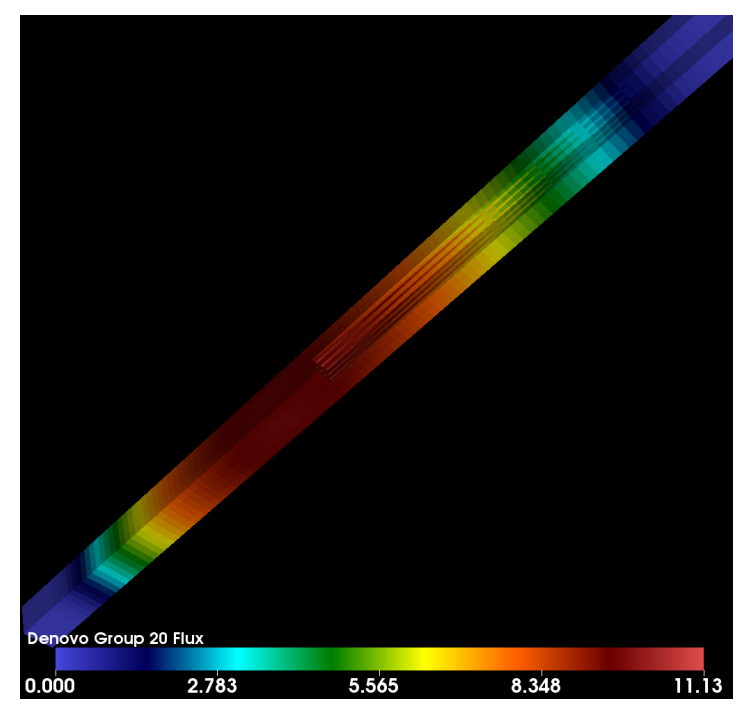

(a) No Scaling

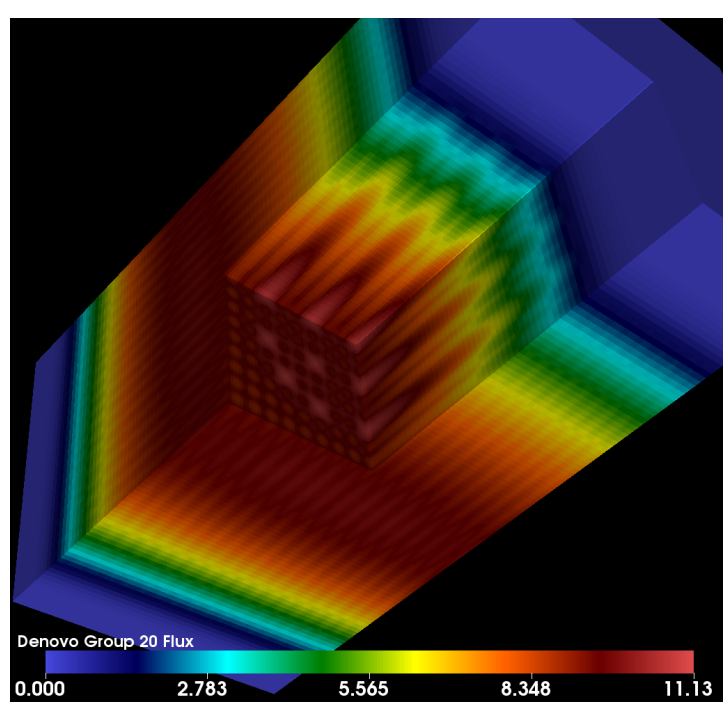

(b) Scaled (10x reduction in height)

Fig. 8. Denovo scalar neutron flux distribution at a resonance energy.

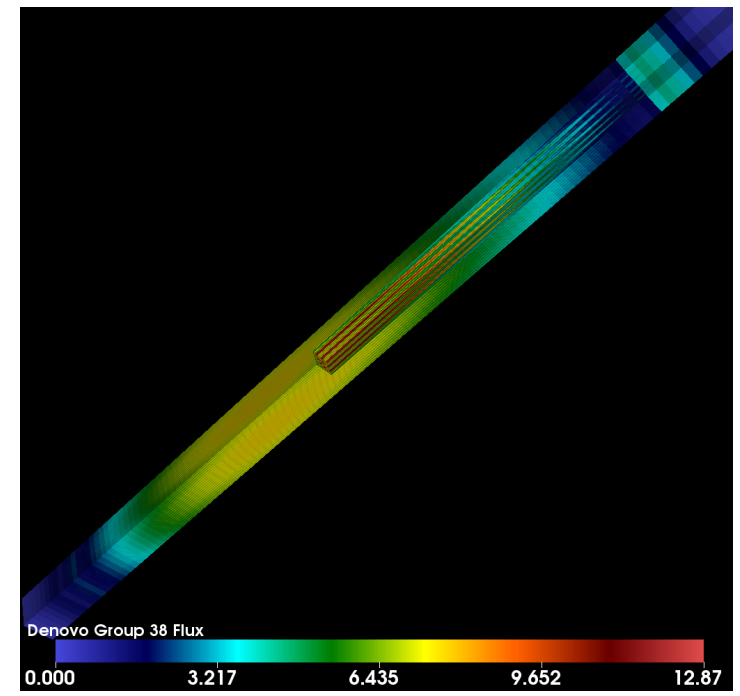

(a) No Scaling

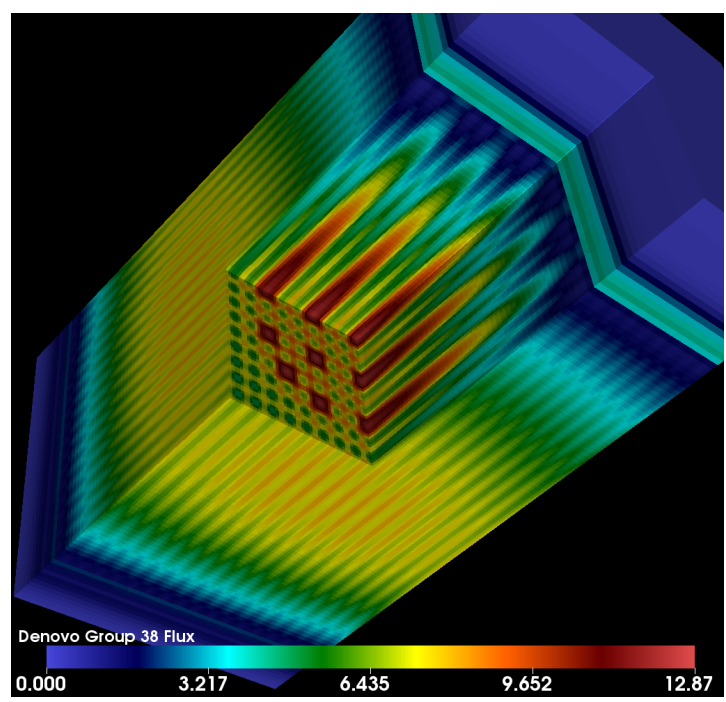

(b) Scaled (10x reduction in height)

Fig. 9. Denovo scalar neutron flux distribution at a thermal energy. 


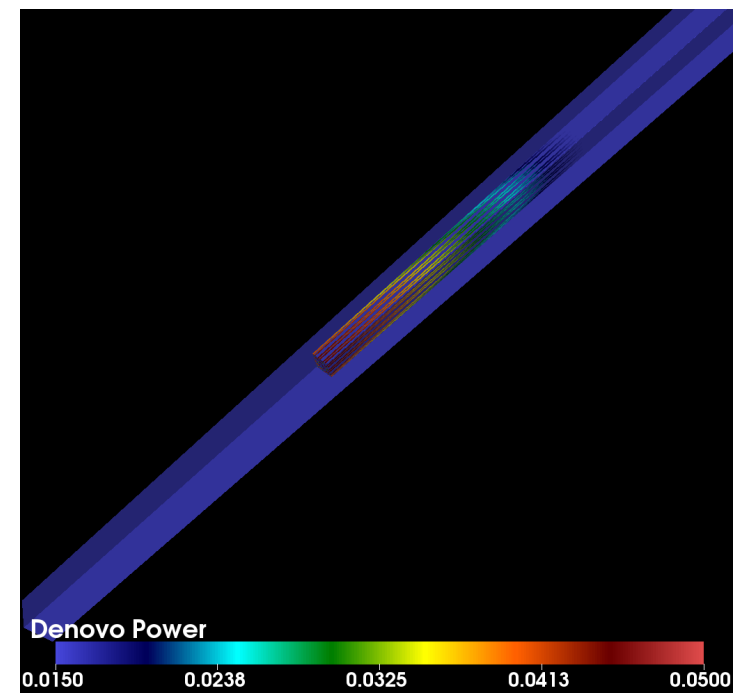

(a) Denovo Power Distribution

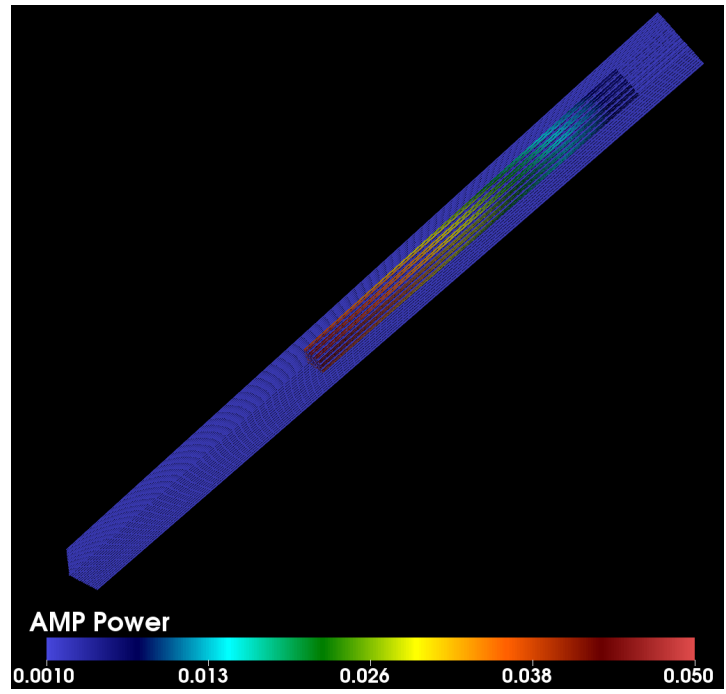

(b) AMPFuel Power Distribution

Fig. 10. Power $\left(\frac{G W}{M T\left(U O_{2}\right)}\right)$ distribution plotted on both the Denovo and AMPFuel meshes.

identical. However, this axial variation would be more significant if additional detail were included, such as grid spacers, mixing vanes, and the effect of inserted control rods or adjacent fuel assemblies that have once- or twice-irradiated fuel assemblies. Therefore, as this problem is expanded to include more fidelity, we anticipate exploring the significance of these assembly features.

The non-axisymmetric variation can be seen in Figure 11 but is more clearly displayed in 2D plots of the power distribution at 3,5,7, and 9 feet from the bottom of the active fuel region (Figures 12 - 15 , respectively). The coarseness of the Denovo solution is apparent in most of these plots but is very clearly seen as lower-power (yellow) regions on the outer edge of the high-power fuel pins, where the Denovo computational cell was only partially filled with fuel. However, the Zernike smoother has eliminated that artifact and provides the temperature and mechanics with a smooth source-term that replicates a much finer Denovo simulation (as discussed in Section 3.1).

Within the assembly, some pins appear to have a relatively axisymmetric power distribution, which justifies the use of 2D (or 1.5D) approximations within traditional fuel performance codes such as FRAPCON. However, the fuel pins that are closer to the guide tubes have a significantly higher power adjacent to the additional moderation, and these are the highest-power pins. There are 100 of 264 pins that are adjacent to guide tubes and 76 that are diagonally adjacent to a guide tube, so two-thirds of all fuel pins have non-axisymmetric power distributions. This variation will lead to non-axisymmetric distributions in the activation and fission products, temperature, distortion, and corrosion.

\subsection{TEMPERATURE DISTRIBUTION}

The temperature distribution within the fuel assembly is shown in three dimensions in Figure 16 . Because the outer radius of the pin is hundreds of degrees cooler than the center, the visualization has the false appearance of being nearly axisymmetric in temperature with little pin-to-pin variation. However, a very small variation in the color represents tens of degrees in temperature variation.

Two-dimensional plots of the temperature distribution at 3, 5, 7, and 9 feet from the bottom of the 


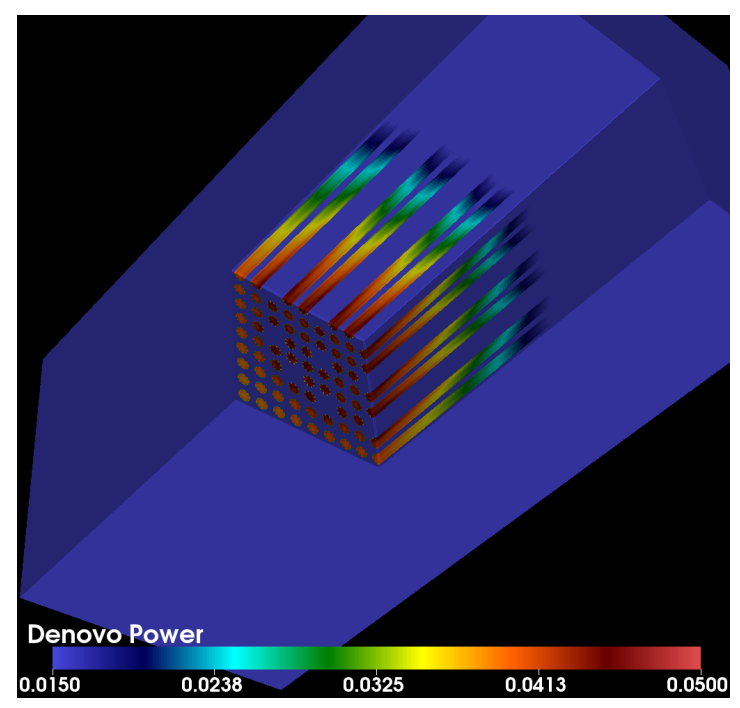

(a) Denovo Power Distribution

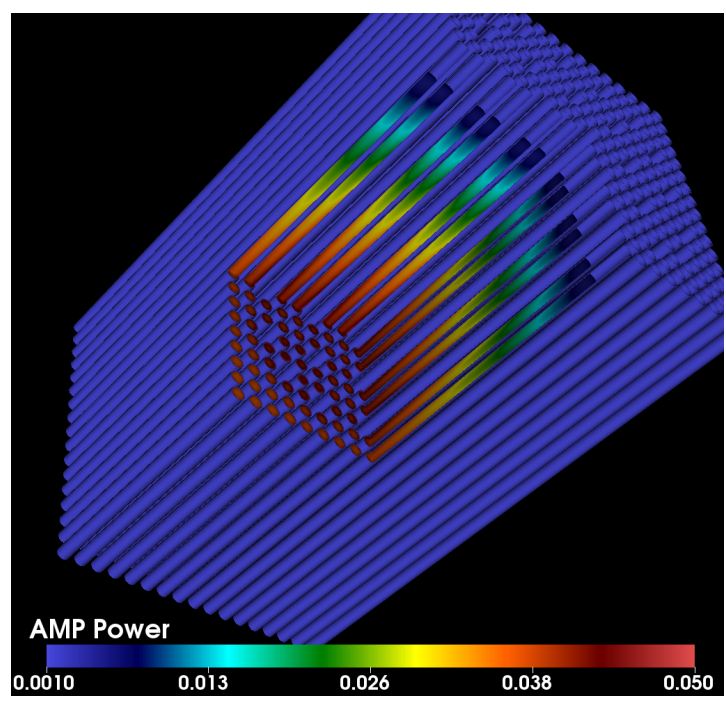

(b) AMPFuel Power Distribution

Fig. 11. Power $\left(\frac{G W}{M T\left(U O_{2}\right)}\right)$ distribution scaled to clearly show the three-dimensional (axial, radial, and azimuthal) variation.

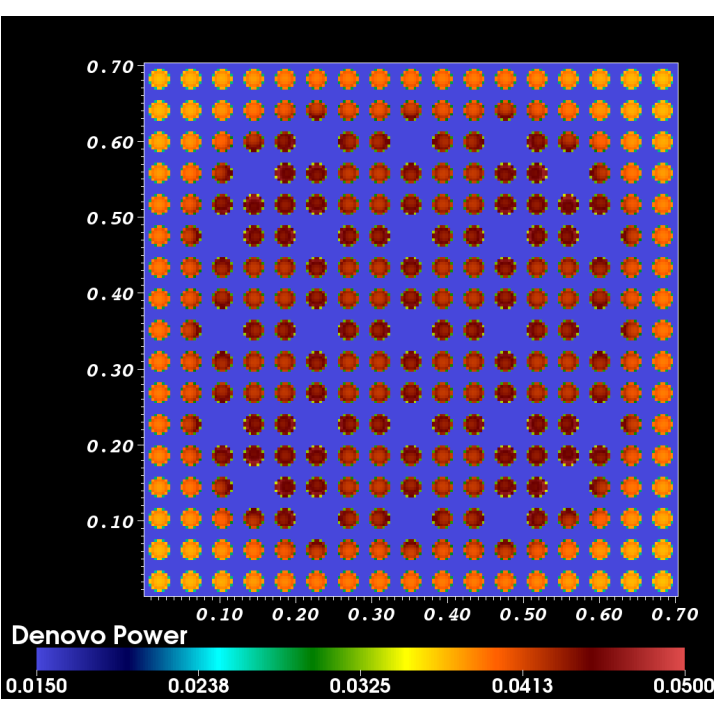

(a) Denovo Power Distribution

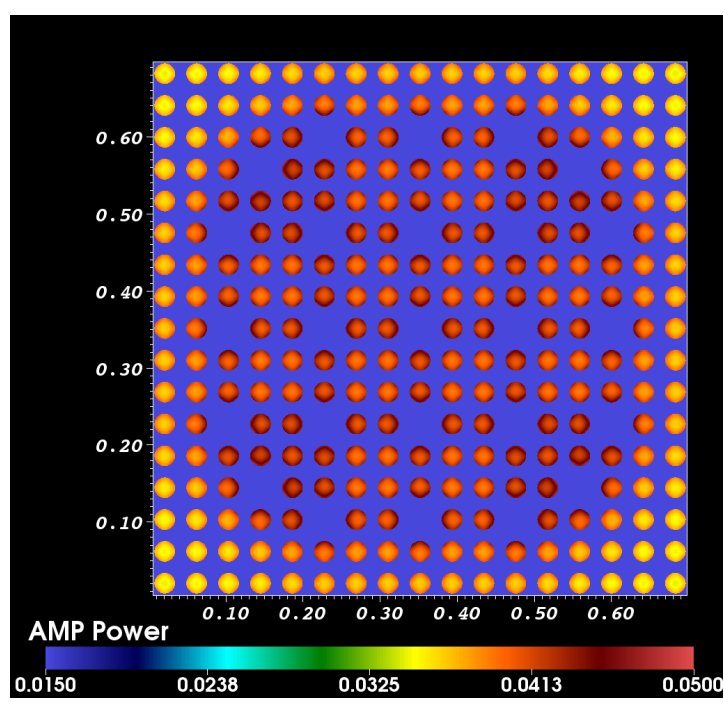

(b) AMPFuel Power Distribution

Fig. 12. Power $\left(\frac{G W}{M T\left(U O_{2}\right)}\right)$ distribution within the assembly 3 feet above the bottom of the active fuel region. 


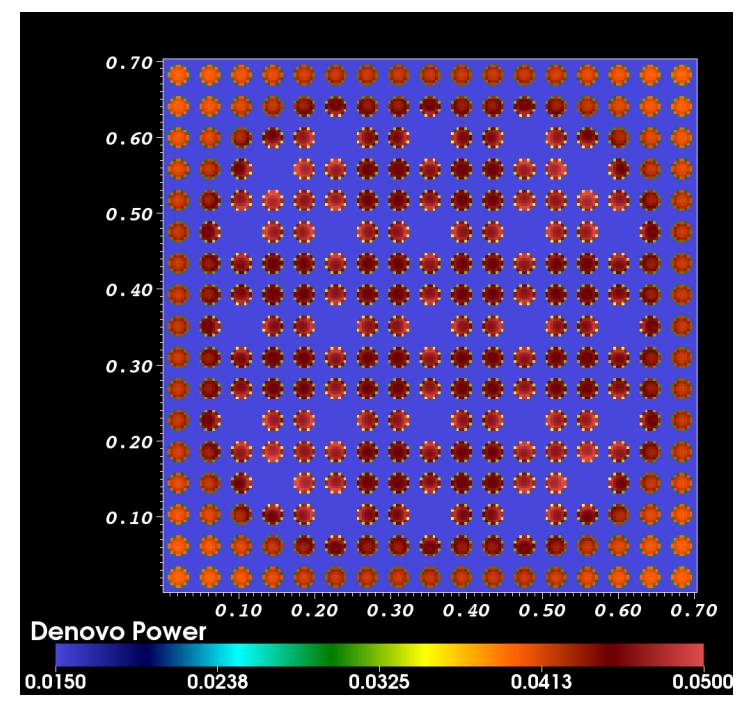

(a) Denovo Power Distribution

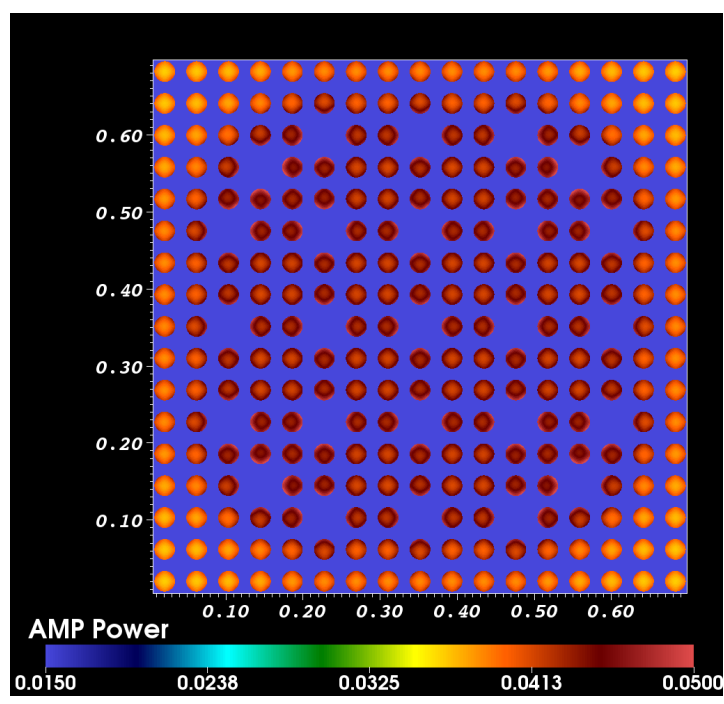

(b) AMPFuel Power Distribution

Fig. 13. Power $\left(\frac{G W}{M T\left(U O_{2}\right)}\right)$ distribution within the assembly 5 feet above the bottom of the active fuel region.

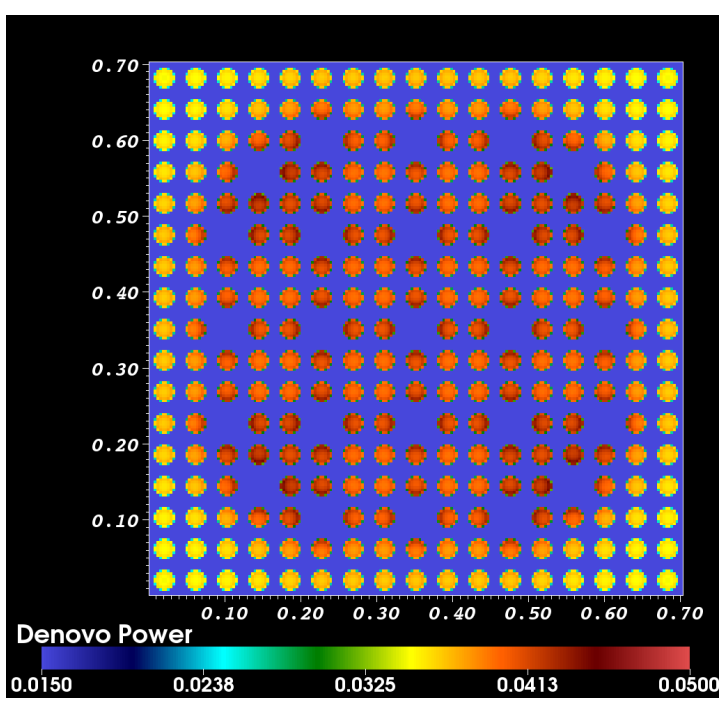

(a) Denovo Power Distribution

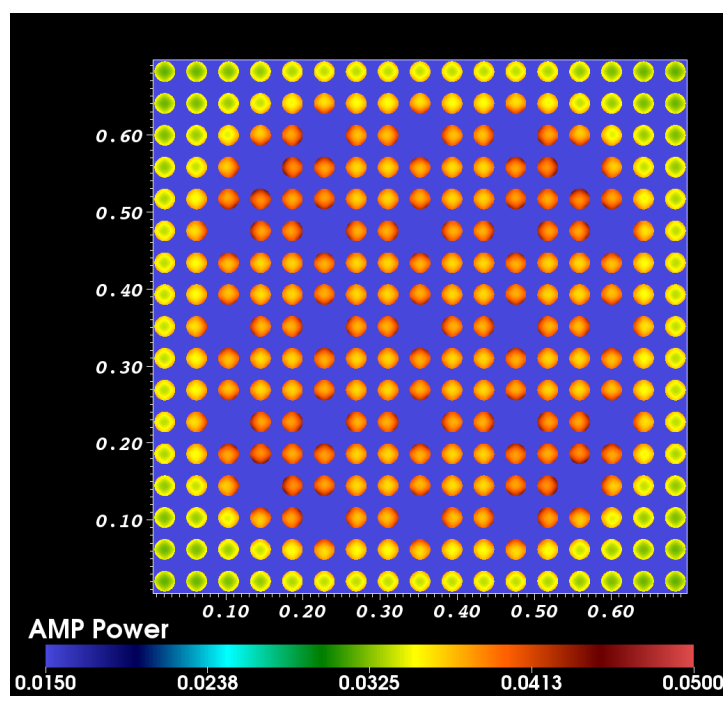

(b) AMPFuel Power Distribution

Fig. 14. Power $\left(\frac{G W}{M T\left(U O_{2}\right)}\right)$ distribution within the assembly 7 feet above the bottom of the active fuel region. 


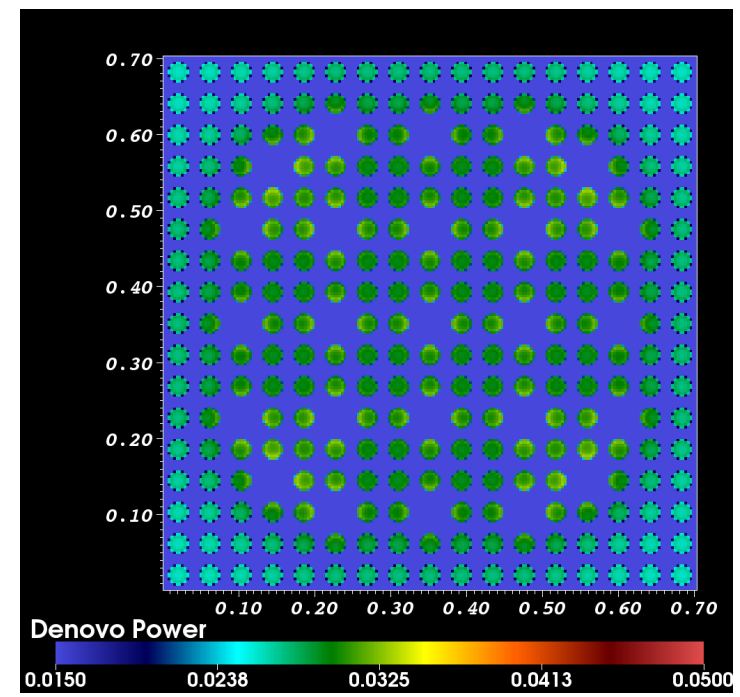

(a) Denovo Power Distribution

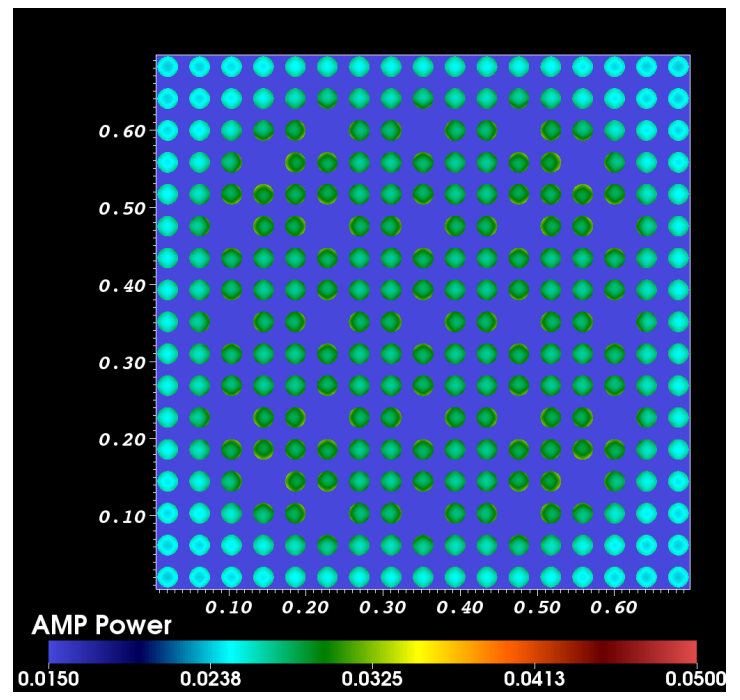

(b) AMPFuel Power Distribution

Fig. 15. Power $\left(\frac{G W}{M T\left(U O_{2}\right)}\right)$ distribution within the assembly 9 feet above the bottom of the active fuel region.

active fuel region are shown in Figure 17. The pin-to-pin power distribution clearly produces variation in fuel pin temperatures, but the effect of the within-pin power distribution is still difficult to ascertain because of the large temperature variation from the cladding surface temperature to the fuel centerline temperature. Three approximations in the boundary conditions prevent concrete observations about the true significance of the (approximately) axisymmetric appearance of the temperature:

- the temperature of the fuel was azimuthally-averaged for use in the cladding Robin boundary condition, and vice versa, which produced a more axisymmetric solution than expected;

- the gap thickness was not used in the model for gap conductance, which produced a less axisymmetric solution than expected; and

- the coolant temperature, which is a boundary condition for the cladding, did not have an azimuthal variation; the effect of which may vary from pin to pin.

The clearest depiction of the significance of the non-axisymmetric temperature distribution can be seen in the mechanical displacement (see Section 4.5) due to thermal expansion, where the (visually-small) azimuthal variation in the temperature causes the fuel regions near the guide tubes to bow. The aforementioned approximations in the boundary conditions will be removed and additional effort in visualization of assembly-scale results will be incorporated in future revisions to better communicate their significance.

\subsection{MECHANICAL STATE}

The total magnitude of the displacement and the magnitude of the axial displacement at the final loading step are shown in Figures 18 and 19 , respectively. In addition, there are expanded views of each at several axial levels in Figures $20-23$ 


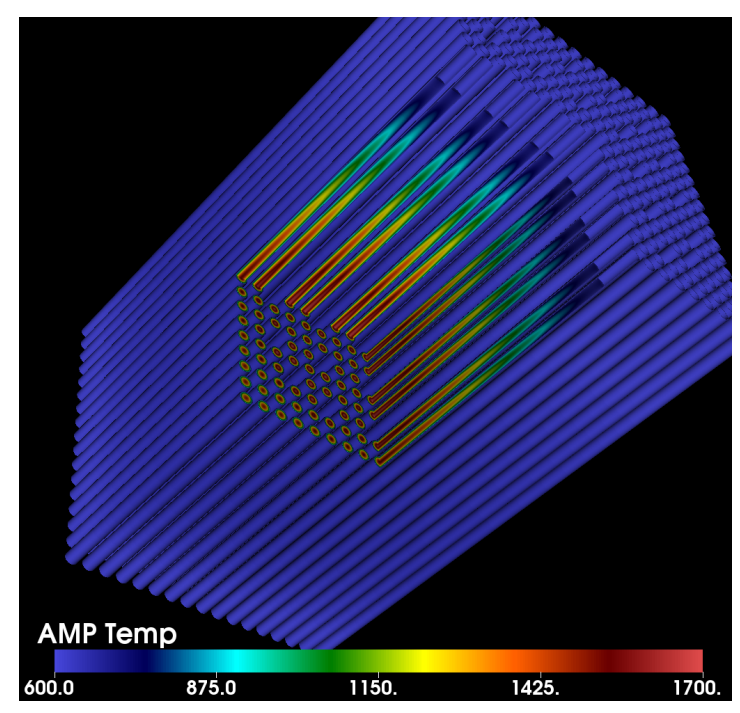

Fig. 16. Temperature (Kelvin) distribution within the assembly.

The maximum axial expansion of the fuel was $1.3 \mathrm{~cm}$, or $0.35 \%$ axial strain, which agrees well with expected results. The axial displacement shows how the axial expansion of the fuel is significantly more than the cladding, which will reduce the free volume in the plenum and increase the pressure of the coolant in gap and effect the fuel-to-clad heat transfer coefficient. In addition, the axial displacement is strongly correlated with the total rod power, which demonstrates the need for accurate pin-to-pin power distributions for predicting fuel performance. In an existing light-water reactor, these power distributions are known to within $1 \%$, but that does not hold for advanced reactors, fuels, or cladding. There should be ample margin in the assembly design that a $1 \%$ error in clad elongation would not reduce the shoulder height and cause the pin to contact the top and bottom assembly plates, which would cause the rod to bow.

The higher (within-pin) power adjacent to guide tubes produces a higher temperature for the entire length of the fuel pin, which leads to increased thermal expansion that produces a "bowing" of the fuel away from the guide tube. This can be seen as a within-pin variation of the axial displacement near the top of the assembly (Figure 22b) but is clear when the total and axial displacement are compared. This is a real effect, but additional investigation is required to understand the effect of boundary conditions at the bottom of the pin, between the fuel regions, and the number of effective fuel regions used. However, it is clear that if the fuel were modeled as a single fuel region (as in many existing fuel performance codes), rather than being treated as independent fuel regions, the bowing would be artificially enhanced because the fuel would bend as a beam and not account for the stress relief between pellets. This is a significant finding because the smearing of the fuel into effective regions was anticipated to be a reasonable approximation to reduce the computational burden when AMPFuel is integrated within Sharp for full reactor simulation.

\section{CONCLUSIONS}

This report describes an initial capability for high-fidelity nuclear fuel assembly simulation and a demonstration of that capability on a full 17×17 PWR assembly. The Advanced Multi-Physics (AMP) Nuclear Fuel Performance code (AMPFuel) includes an integrated nuclear fuel assembly capability for (one-way) coupled radiation transport and nuclear fuel assembly thermo-mechanics. AMPFuel leverages 


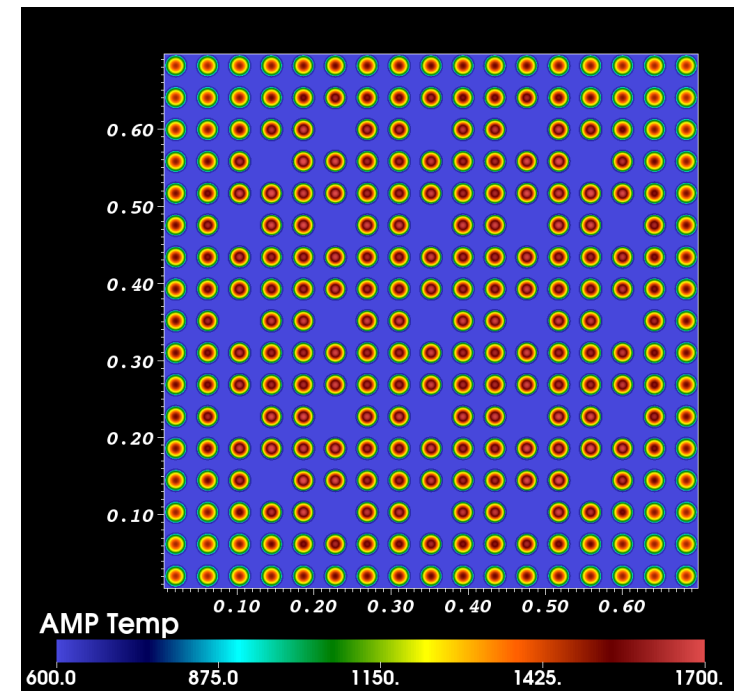

(a) 3 feet

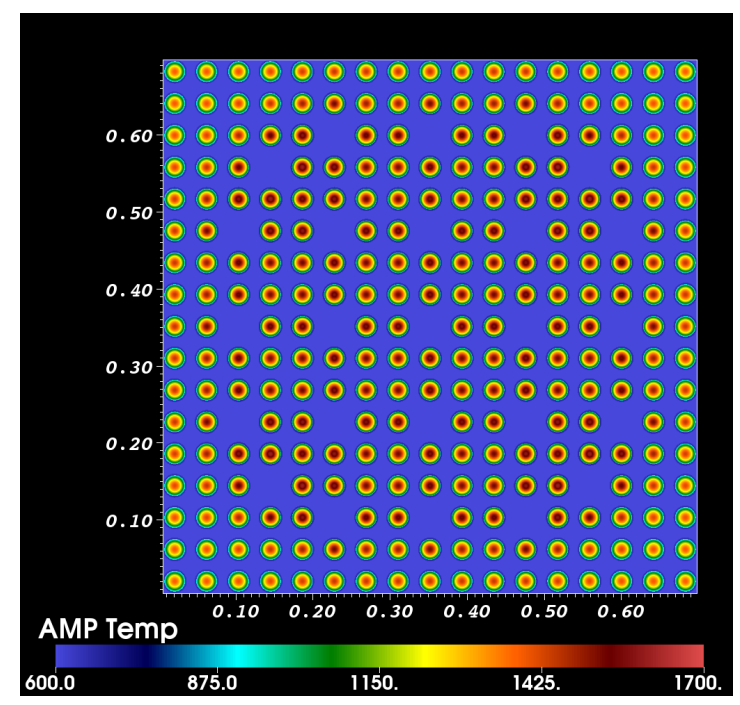

(c) 7 feet

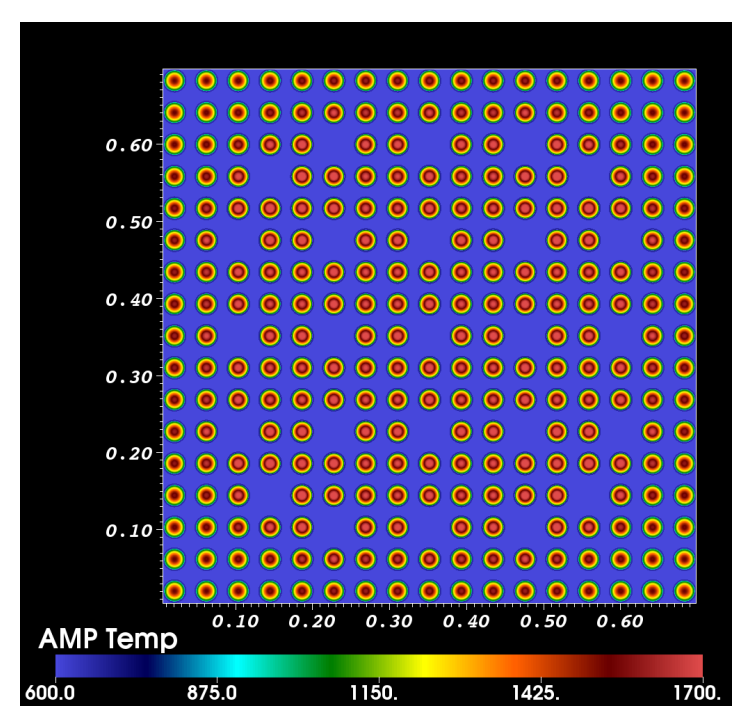

(b) 5 feet

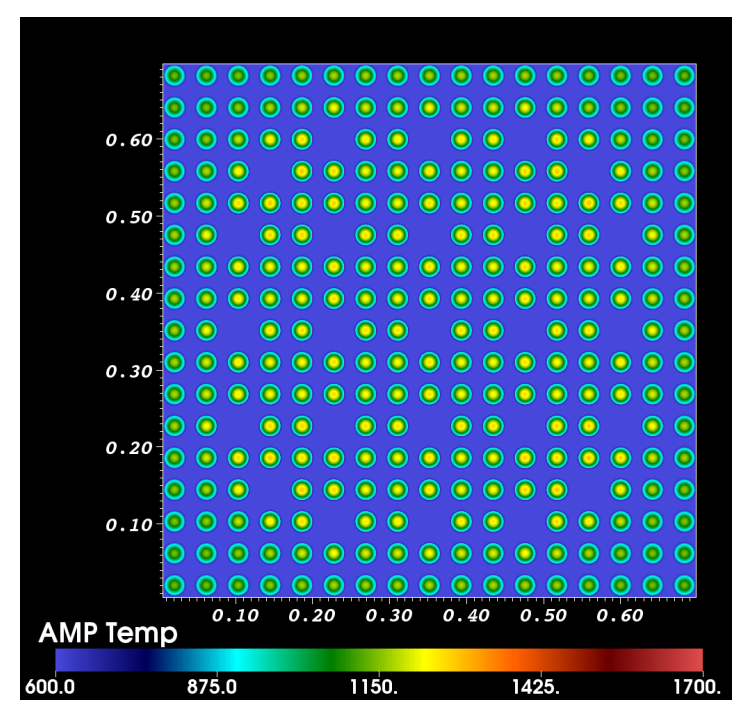

(d) 9 feet

Fig. 17. Temperature (Kelvin) distribution within the assembly for various heights above the bottom of the active fuel region. 


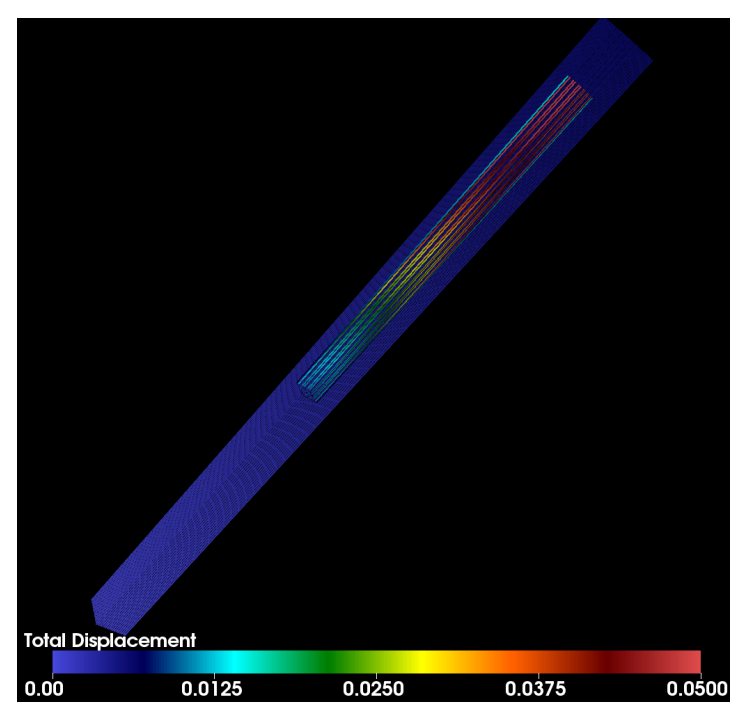

(a) No scaling

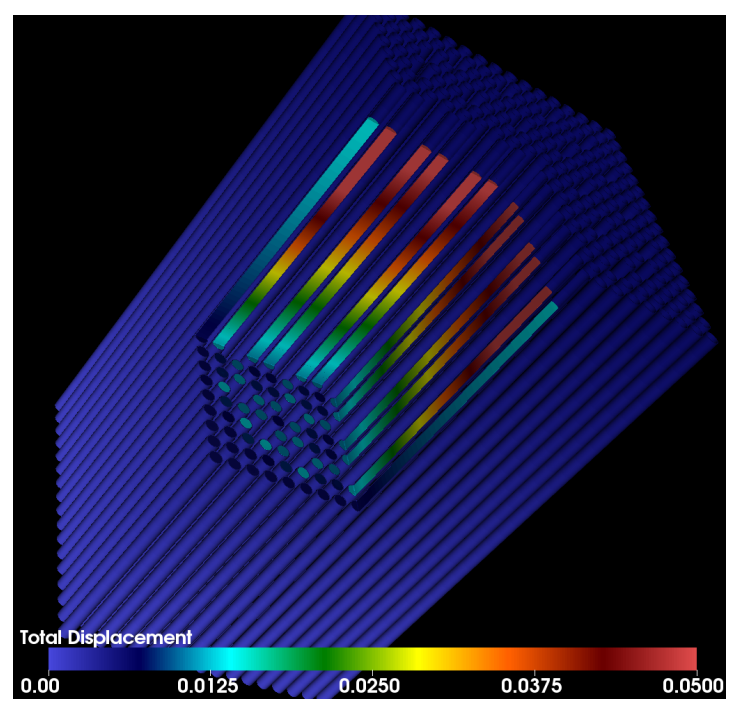

(b) Scaled (10x reduction in height)

Fig. 18. Total displacement magnitude (meters) on the initial AMPFuel mesh.

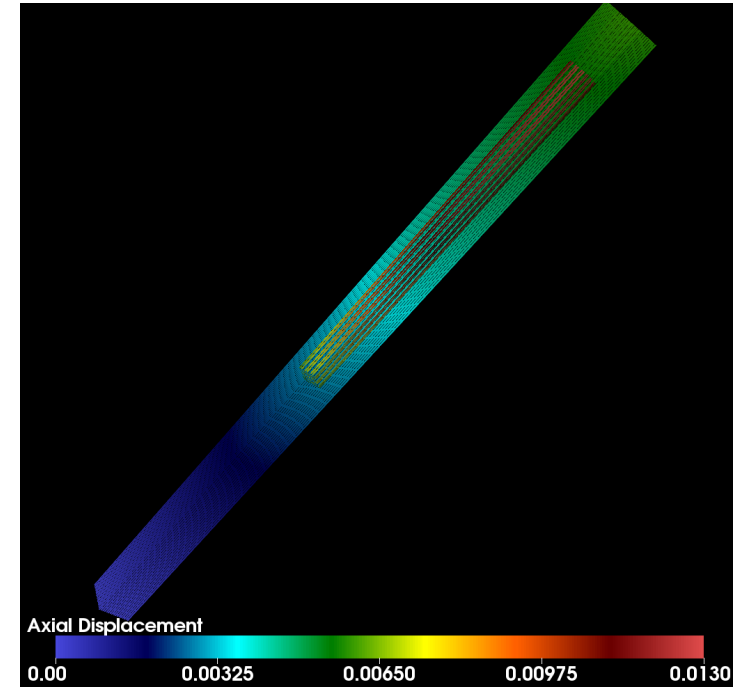

(a) No scaling

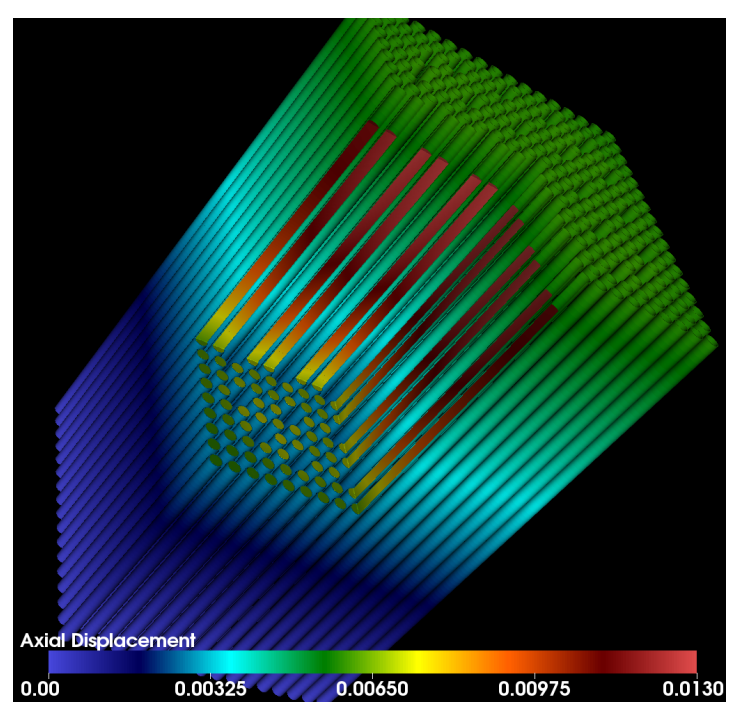

(b) Scaled (10x reduction in height)

Fig. 19. Axial displacement (meters) on the initial AMPFuel mesh. 


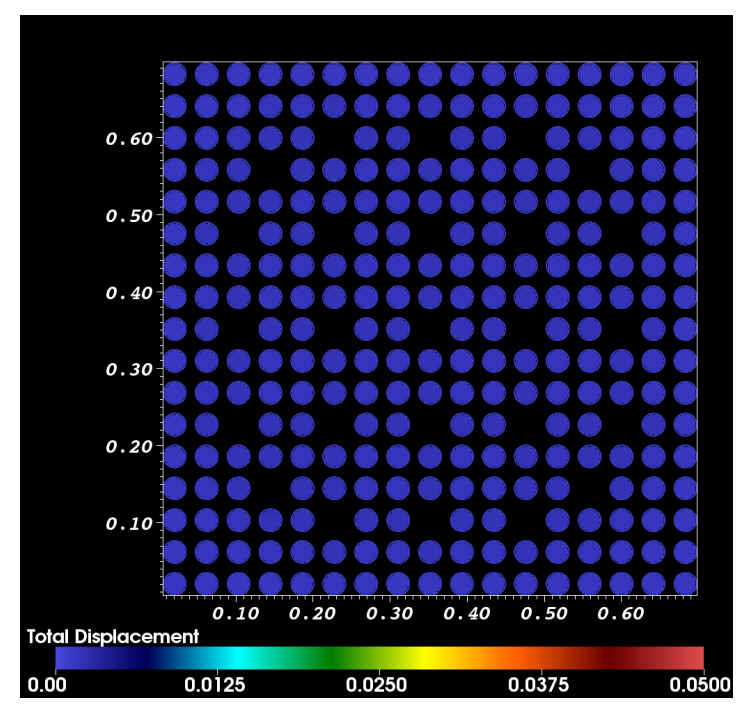

(a) Total

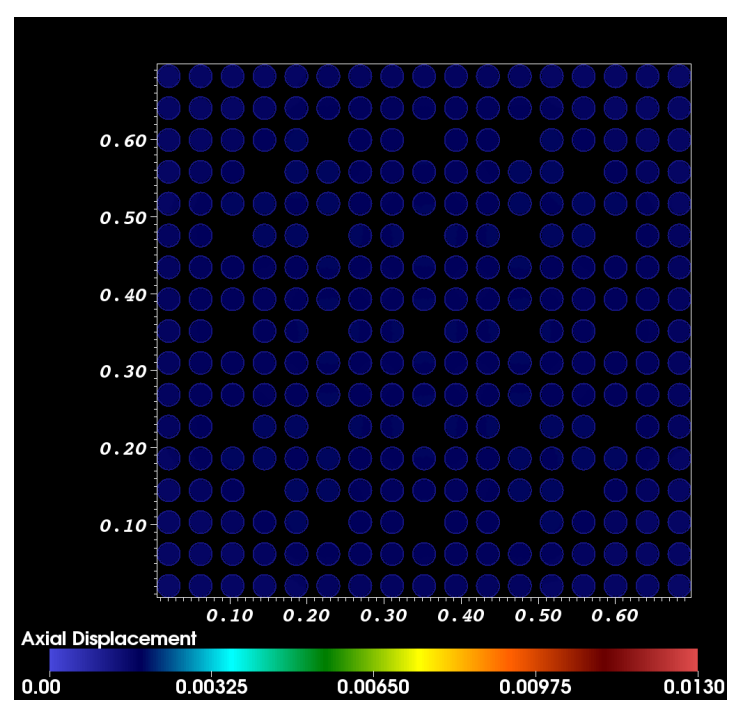

(b) Axial

Fig. 20. Total and axial displacement (meters) within the assembly 3 feet above the bottom of the active fuel region.

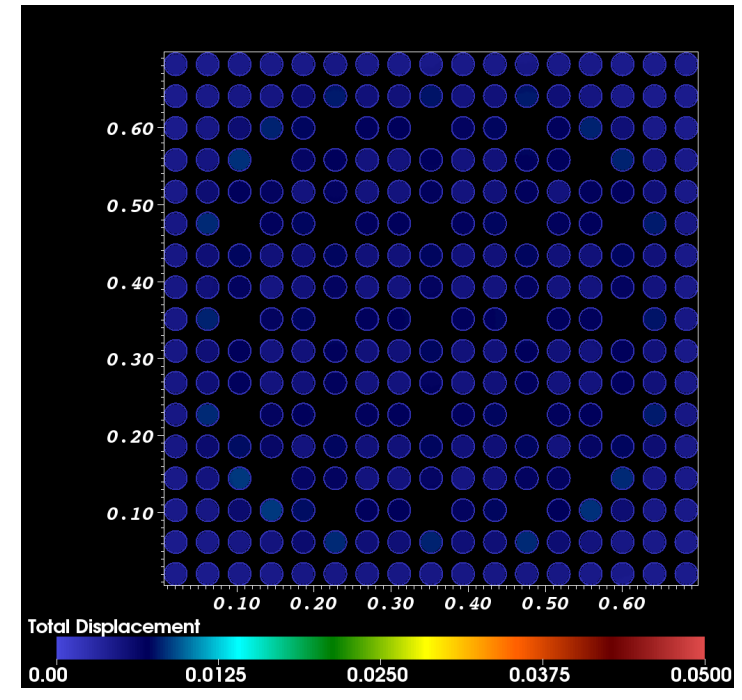

(a) Total

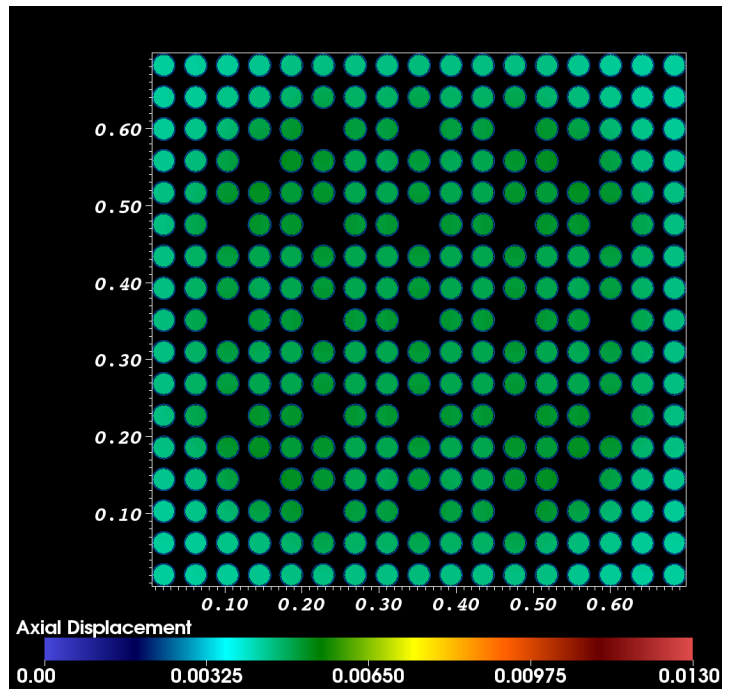

(b) Axial

Fig. 21. Total and axial displacement (meters) within the assembly 5 feet above the bottom of the active fuel region. 


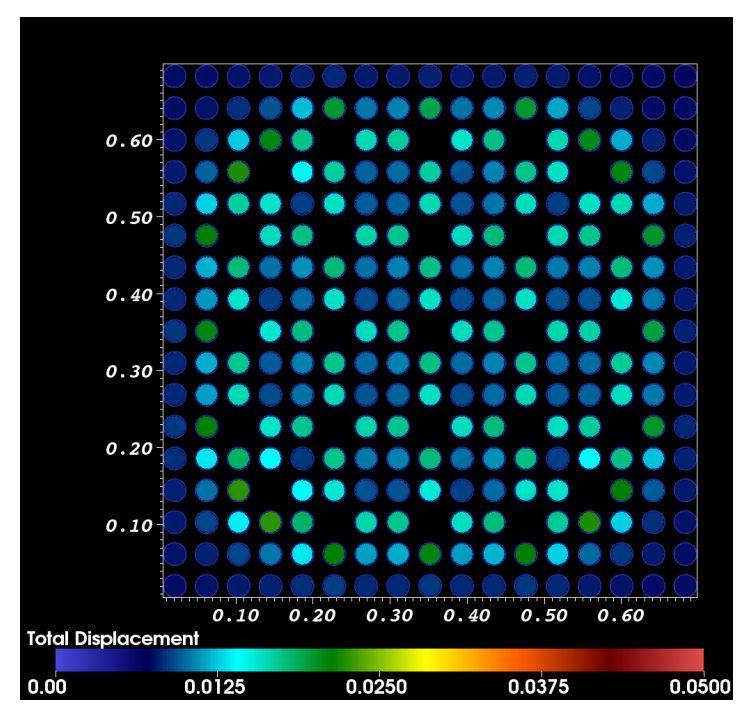

(a) Total

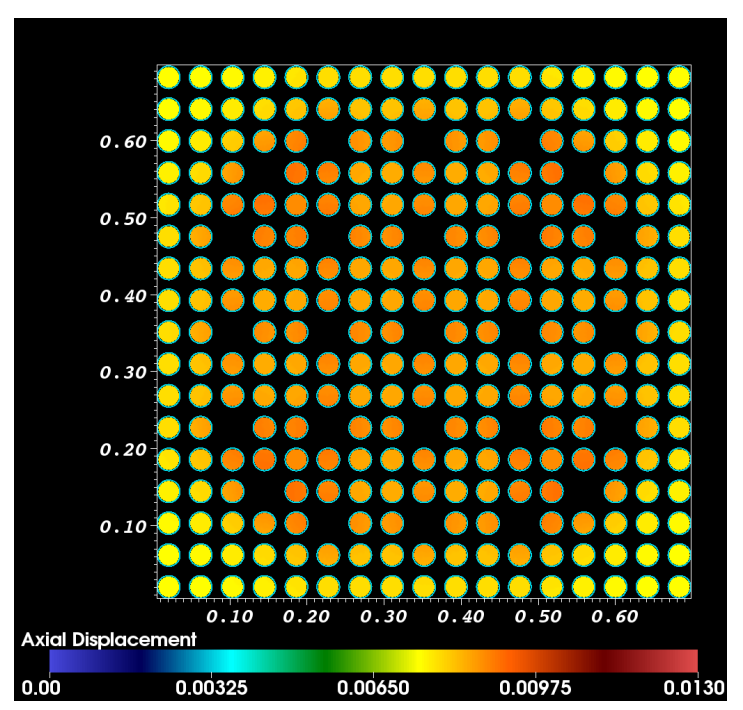

(b) Axial

Fig. 22. Total and axial displacement (meters) within the assembly 7 feet above the bottom of the active fuel region.

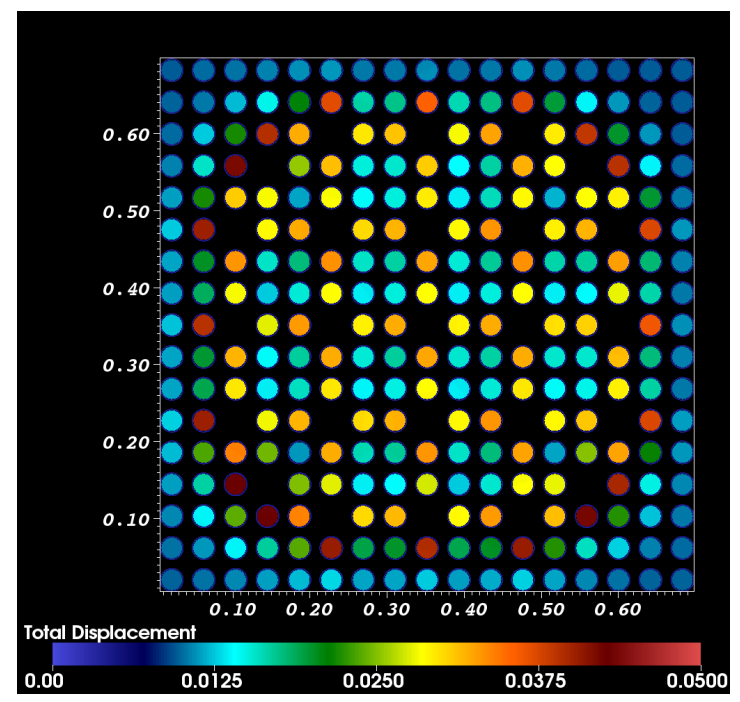

(a) Total

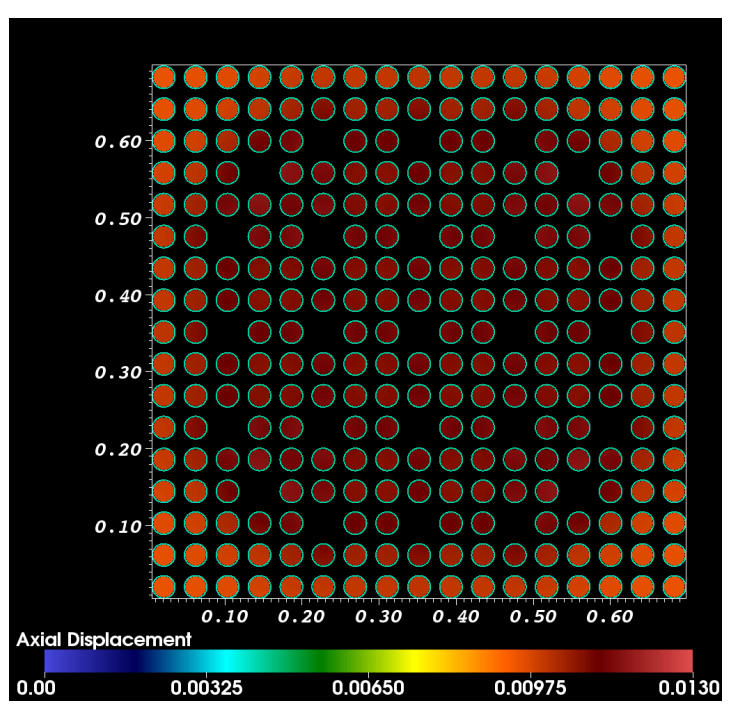

(b) Axial

Fig. 23. Total and axial displacement (meters) within the assembly 9 feet above the bottom of the active fuel region. 
the Scale/Denovo radiation transport code to provide a multi-group, discrete-ordinates, k-eigenvalue solution to the first-order form of the Boltzmann transport equation, which defines the spatial distribution of the heat source (power) within the problem. The heat transfer and mechanics are discretized with the linear finite-element formulation of the diffusion and elastic-plastic mechanics equations, with appropriate material models and boundary conditions, and each is solved with a fully-coupled Jacobian-Free Newton Krylov solver that leverages both algebraic multi-grid and a physics-based mechanics preconditioner. Both the Denovo and thermo-mechanics simulations were executed in parallel and leveraged a domain decomposition designed for nuclear fuel assembly simulations that minimized communication between domains.

The integrated assembly simulation capability was used to model a representative fuel assembly with many of the features resolved in three dimensions. The full problem definition included the fuel, gap, and cladding of each of the 264 fuel pins; the 25 guide tubes; the top and bottom structural regions; and the upper and lower (neutron) reflector regions. Both AMPFuel and Denovo include some simplifications of the geometry, which required careful coordination, but provided an integrated solution to the multi-group neutron flux, fission power distribution, temperature distribution (in the fuel, gap, and cladding), and mechanical deformation of the fuel and cladding. However, both AMPFuel and Denovo include embedded mesh generation with Python-based input generation routines, which allows for easy problem setup and execution (relative to codes that require a complex CAD [computer-aided design] model of the entire domain and user-intensive meshing tools).

A novel scheme was developed for transferring the power distribution from Denovo (structured, Cartesian mesh with smeared materials within each cell) to AMPFuel (unstructured, hexagonal mesh with a single material within each cell). The approach leveraged the geometry of the problem (cylindrical) to utilize an orthogonal basis in the radial, azimuthal, and axial dimensions to ensure that the power from Denovo was transferred with an appropriate smoothing function that significantly reduced the effect of artificial discontinuities in the power for use in the AMPFuel thermo-mechanics calculations. This allowed the use of a relatively coarse spatial mesh for the radiation transport (44 groups and 128 directions per cell) and a fine spatial mesh for thermo-mechanics (4 DOF per node) with very little loss of accuracy. In addition, the high aspect ratio of each fuel pin (12 ft axially, but $\frac{1}{4}$ in. in diameter) presented a challenge for standard Algebraic Multi-Grid (AMG) techniques for mechanics and required the development of a new nuclear fuel-specific preconditioner. The new preconditioner allows for the use of AMG on each individual fuel region as an inner preconditioner while accounting for their cumulative effect on the axial elongation with an outer (Krylov) iteration.

Mesh refinement and timing studies were performed on a limited set of problems (thermal-only) to evaluate the relative computational performance and accuracy of the mesh-to-mesh solution transfer of AMPFuel and Denovo. The final, full-assembly calculation was executed on Jaguar using 40,000 cores in under 10 hours to model over 162 billion DOF for 10 loading steps. The single radiation transport calculation required about $50 \%$ of the time required to solve the thermo-mechanics with a single loading step, which demonstrates that it is feasible to incorporate, in a single code, a high-fidelity radiation transport capability with a high-fidelity nuclear fuel thermo-mechanics capability and anticipate acceptable computational requirements. If a single mesh had been used, the transport would have been at least 50 times larger and more than $98 \%$ of the computational time.

The results of the full-assembly simulation clearly show the axial, radial, and azimuthal variation of the neutron flux, power, temperature, and deformation of the assembly, highlighting behavior that is neglected in traditional axisymmetric fuel performance codes. The multi-group neutron flux distribution captures the spatial distribution due to both assembly features and resonance self-shielding that will lead to a "rim 
effect" with depletion. Because it is based on 3D radiation transport, rather than calibrated models, it is extensible to incorporate additional assembly features, such as grid spacers and part-length rods, that create additional heterogeneity in the flux and power distribution. The multidimensional multi-group neutron flux distribution led to a power distribution between pins and within pins that demonstrated the regions of the assembly where axisymmetric fuel modeling is appropriate, but also displayed the missing effects in the highest-power fuel pins, which are adjacent to guide tubes. This non-axisymmetric power distribution created a non-axisymmetric temperature distribution, which produced a rod bowing effect due to increased thermal expansion near the guide tubes.

As this problem is extended to include more assembly features, different reactor and fuel types, reduced approximations in the thermo-mechanics, coupling of the physics, and isotopic depletion, we will be able to develop a more comprehensive understanding of the physics and models at a resolution that was previously unattainable and develop approximations that can improve the accuracy of reactor simulations (which use lower-fidelity fuel models) and single-pin fuel simulations (which use lower fidelity neutronics models).

\section{ACKNOWLEDGMENTS}

The development of the AMPFuel code was funded by the Nuclear Energy Advanced Modeling and Simulation program of the US Department of Energy Office of Nuclear Energy, Advanced Modeling and Simulation Office. This project acknowledges support for Mark Berrill from the Eugene P. Wigner Fellowship at Oak Ridge National Laboratory, managed by UT-Battelle, LLC, for the US Department of Energy under Contract DE-AC05-00OR22725, and for James Banfield using funding received through a grant to the University of Tennessee-Knoxville from the DOE Office of Nuclear Energy's Nuclear Energy University Programs.

Computational resources were provided through a US Department of Energy INCITE (Innovative and Novel Computational Impact on Theory and Experiment) Award: "Uncertainty Quantification for Three-Dimensional Reactor Assembly Simulations."

The AMPFuel is available, with limited distribution, through the Radiation Safety Information Computational Center (RSICC) at Oak Ridge National Laboratory as BCC-001. 


\section{References}

[1] SCALE: A modular code system for performing standardized computer analyses for licensing evaluation. Technical Report ORNL/TM-2005/39, Version 6, Vols. I - III, Oak Ridge National Laboratory, Oak Ridge, Tennessee, USA, 2005. Available from Radiation Safety Information Computational Center at Oak Ridge National Laboratory as CCC-750, http://www. ornl.gov/sci/scale/.

[2] M. Asgari, T. Bahadir, D. Kropaczek, E. Gibson, and J. Williams. Analysis of the pin power peaking of the Hatch unit 1 cycle 21 failed fuel assemblies. In Proceedings of the 2010 LWR Fuel Performance/TopFuel/WRFPM, Orlando, FL, September 2010.

[3] G. A. Berna, C. E. Beyer, K. L. Davis, and D. D. Lanning. FRAPCON-3: A computer code for the calculation of steady-state, thermal-mechanical behavior of oxide fuel rods for high burnup. Technical Report NUREG/CR-6534, Pacific Northwest National Laboratory, 1997.

[4] K.T. Clarno, B. Philip, W.K. Cochran, R.S. Sampath, S. Allu, P. Barai, S. Simunovic, L.J. Ott, S. Pannala, P. Nukala, G. Dilts, B. Mihaila, C. Unal, G. Yesilyurt, J.H. Lee, J.E. Banfield, and G.I. Maldonado. The AMP (Advanced MultiPhysics) Nuclear Fuel Performance code. Technical Report ORNL/LTR-2011/42, Oak Ridge National Laboratory, Oak Ridge, TN, USA, February 2011.

[5] G.G. Davidson, T.M. Evans, R.N. Slaybaugh, and C.G. Baker. Massively parallel solutions to the k-eigenvalue problem. Trans. Am. Nucl. Soc., 81:318-320, 2010.

[6] T.M. Evans, A.S. Stafford, R.N. Slaybaugh, and K.T. Clarno. Denovo: A new three-dimensional parallel discrete ordinates code in SCALE. Nuclear Technology, 171:171-200, 2010.

[7] S.P. Hamilton and K.T. Clarno. Mathematical framework for the coupling of the AMP and Denovo codes. Trans. Am. Nucl. Soc., 2011.

[8] Michael Heroux, Roscoe Bartlett, Vicki Howle Robert Hoekstra, Jonathan Hu, Tamara Kolda, Richard Lehoucq, Kevin Long, Roger Pawlowski, Eric Phipps, Andrew Salinger, Heidi Thornquist, Ray Tuminaro, James Willenbring, and Alan Williams. An overview of Trilinos. Technical Report SAND2003-2927, Sandia National Laboratories, 2003.

[9] K.R. Koch, R.S. Baker, and R.E. Alcouffe. A parallel algorithm for 3D $S_{n}$ transport sweeps. Technical report, LA-CP-92-406, Los Alamos National Laboratory, 1992.

[10] D. D. Lanning, C. E. Beyer, and K. J. Geelhood. FRAPCON-3 updates, including mixed-oxide fuel properties. Technical Report NUREG/CR-6534, Pacific Northwest National Laboratory, 2005.

[11] E. W. Larsen. Unconditionally stable diffusion-acceleration of the transport equation. Transport Theory and Statistical Physics, 11:29, 1982.

[12] K. Lassmann. The radial distribution of plutonium in high burnup $\mathrm{UO}_{2}$ fuels. Journal of Nuclear Materials, 188:295, 1992.

[13] K. Lassmann, C. O'Carroll, J. van de Laar, and C.T. Walker. The radial distribution of plutonium in high burnup $\mathrm{UO}_{2}$ fuels. Journal of Nuclear Materials, 208:223-231, 1994. 
[14] L.J. Ott, T. Tverberg, and E. Sartori. Summary of irradiation tests of mixed oxide fuel prepared with weapons-derived plutonium. In Proc. of PHYSOR 2008 International Conference on the Physics of Reactors Nuclear Power: A Sustainable Resource, Las Vegas, NV, September 2008.

[15] A. Schubert, P. Van Uffelen, J. Van de Laar, C.T. Walker, and W. Haeck. Extension of the TRANSURANUS burn-up model. Journal of Nuclear Materials, 376:1-10, 2008.

[16] G. Yesilyurt, K.T. Clarno, T.M. Evans, G.G. Davidson, and P.B. Fox. C5 benchmark problem with discrete ordinate radiation transport code Denovo. Nuclear Science and Engineering, 176:274 - 283, 2011. 